\title{
Knot theory with the Lorentz group
}

\author{
by
}

João Faria Martins (Lisboa)

\begin{abstract}
We analyse perturbative expansions of the invariants defined from unitary representations of the Quantum Lorentz Group in two different ways, namely using the Kontsevich Integral and weight systems, and the $R$-matrix in the Quantum Lorentz Group defined by Buffenoir and Roche. The two formulations are proved to be equivalent; and they both yield $\mathbb{C}[[h]]$-valued knot invariants related with the Melvin-Morton expansion of the Coloured Jones Polynomial.
\end{abstract}

Introduction. The main aim of this article is to show a possible path to define knot invariants from infinite-dimensional representations of the Lorentz group.

Let $\mathcal{A}$ be a Hopf algebra; its category of finite-dimensional representations is a compact monoidal category. Let $q$ be a complex number not equal to 1 or -1 . Suppose $\mathcal{A}=U_{q}(\mathfrak{g})$ is the Drinfeld-Jimbo algebra attached to the semisimple Lie algebra $\mathfrak{g}$. Even though $\mathcal{A}$ is not a ribbon Hopf algebra, it possesses a formal $R$-matrix and a formal ribbon element. These elements make sense when applied to finite-dimensional representations of $\mathcal{A}$, and thus its category of finite-dimensional representations is a ribbon category. This means we have a knot invariant attached to any finite-dimensional representation of $\mathcal{A}$. This kind of knot invariants take values in $\mathbb{C}$.

A similar situation happens in the case of the Quantum Lorentz Group $\mathcal{D}$ as defined by Woronowicz and Podleś in [PoW]. We shall use especially the further developments of its theory by Buffenoir and Roche (see [BR1] and [BR2]). Despite the fact $\mathcal{D}$ is not a Drinfeld-Jimbo algebra, its structure of a quantum double, namely $\mathcal{D}=\mathcal{D}\left(U_{q}(\mathfrak{s u}(2)), \operatorname{Pol}\left(S U_{q}(2)\right)\right)$ with $q \in(0,1)$, makes possible the definition of a formal $R$-matrix on it. Also, it is possible to define a heuristic ribbon element.

2000 Mathematics Subject Classification: 57M27, 17B37, 20G42.

Key words and phrases: Kontsevich Integral, Coloured Jones Polynomial, Quantum Lorentz Group. 
The category of finite-dimensional representations of $\mathcal{D}$ can be proved to be a ribbon category, and thus we can define knot invariants from it. In fact, as observed in [BR2], it is possible to prove that this category is ribbon equivalent to the category of finite-dimensional representations of $U_{q}(\mathfrak{s u}(2)) \otimes_{R^{-1}} U_{q}(\mathfrak{s u}(2))$. This last bialgebra equals $U_{q}(\mathfrak{s u}(2)) \otimes U_{q}(\mathfrak{s u}(2))$ as an algebra but has a coproduct twisted by $R^{-1}$, the inverse of the $R$ matrix of $U_{q}(\mathfrak{s u}(2))$. This equivalence relates the knot invariants obtained to the Coloured Jones Polynomial in a nice way.

Such a splitting of $\mathcal{D}$ is not, however, the most natural when considering unitary infinite-dimensional representations of $\mathcal{D}$. The general classification of the unitary representations of the Quantum Lorentz Group is due to Pusz (cf. $[\mathrm{Pu}]$ ). In this case, as well as in the case of harmonic analysis, its definition as a quantum double is usually easier to deal with. As observed in [BR1], it is possible to describe the action of the formal $R$-matrix of the Quantum Lorentz Group in a class of infinite-dimensional representations of that group. For this reason, it is natural to ask whether there exists a knot theory attached to the infinite-dimensional representations of $\mathcal{D}$ (see also [G]).

We shall see the answer is affirmative at least on the perturbative level. Since we are working with infinite-dimensional representations, the general approach of Reshetikhin and Turaev for constructing knot invariants cannot be directly applied. It is possible, though, given a knot diagram, or to be more precise a connected $(1,1)$-tangle diagram, to make a heuristic evaluation of the Reshetikhin-Turaev functor on it. This yields an infinite series for any knot diagram. This method was also elucidated in $[\mathrm{NR}]$. Unfortunately, at least for unitary infinite-dimensional representations, these infinite series do not seem to converge at least for some simple knot diagrams. However, they converge $h$-adically for $q=\exp (h / 2)$, since the order of expansion of their terms as a power series in $h$ is increasing (where the order of a power series is the degree of the first non-zero term). Therefore these evaluations do define $\mathbb{C}[[h]]$-valued knot invariants. This article aims to define these invariants from the Kontsevich Integral and weight systems.

Let $\mathfrak{g}$ be a semisimple Lie algebra. The $h$-adic variant of Drinfeld-Jimbo algebras, that is, the algebras $U_{h}(\mathfrak{g})$, is usually more practical to deal with if one wants to define knot invariants from infinite-dimensional representations of $\mathfrak{g}$. Let $K$ be a given knot. The fact that $U_{h}(\mathfrak{g})$ is a ribbon Hopf algebra, and not merely a formal ribbon Hopf algebra, makes it possible to define a central element of the algebra from $K$, or to be more precise from a 2-dimensional diagram of it. See for example [LM]. This central element is well defined and is a knot invariant. The centre of $U_{h}(\mathfrak{g})$ is isomorphic, through a canonical isomorphism, to the algebra of formal power series on the centre of the universal enveloping algebra $U(\mathfrak{g})$ of $\mathfrak{g}$. This means that given a Lie algebra $\mathfrak{g}$ we have a knot invariant taking its values in the 
algebra of formal power series over the centre of $U(\mathfrak{g})$. This invariant can be described by means of the Kontsevich Integral. If we have an irreducible finite-dimensional representation $V$ of $\mathfrak{g}$, each of the terms of the formal power series associated with the knot $K$ will then act in $V$ as a multiple of the identity. Therefore we can transform a formal power series on the centre of $U(\mathfrak{g})$ into a formal power series over $\mathbb{C}$. If the representations are finite-dimensional, these power series have a non-zero radius of convergence and their value at $h=2 \log (q)$ is the value of the (rescaled) knot invariant associated with $U_{q}(\mathfrak{g})$, as long as we use the representation of $U_{q}(\mathfrak{g})$ that quantises the representation $V$ of $\mathfrak{g}$ with which we are working.

Notice that nothing prevents the same framework from being applied to an infinite-dimensional representation of $\mathfrak{g}$, as long as any central element of $U(\mathfrak{g})$ acts in it as a multiple of the identity. Representations of this kind appear frequently in Lie algebra theory, and are commonly known as representations which admit a central character. Examples are the irreducible cyclic highest weight representations of $\mathfrak{g}$, for $\mathfrak{g}$ semisimple, which, in the $\mathfrak{s l}(2, \mathbb{C})$ context, are simply constructed by perturbing the spin representation in such a way that we admit arbitrary complex spins. In this case this yields a knot invariant which is in some sense an analytic continuation of the Coloured Jones Polynomial.

Other examples of infinite-dimensional representations that admit a central character are the representations of the Lie algebra $L$ of the Lorentz Group which correspond to the representations of the Lorentz Group in the principal series. These are the classical counterpart of the representations of the Quantum Lorentz Group considered in [BR2]. Therefore we would expect the knot power series invariants that result from their use to relate somehow to the knot invariants that come from infinite-dimensional representations of the Quantum Lorentz Group.

One of the main results of this article (Theorem 25) supports these expectations. Of course the way we construct central elements of the enveloping algebra of $L$ must be specified. Notice that the Quantum Lorentz Group is not the Drinfeld--Jimbo algebra associated with the Lorentz Algebra $L$. For the reasons pointed out before, one solution is to define the $h$-adic quantised universal enveloping algebra of $L$ in a non-standard way as $U_{h}(\mathfrak{s u}(2)) \otimes_{R^{-1}} U_{h}(\mathfrak{s u}(2))$. Another solution, which is equivalent, is to use the Kontsevich Universal Knot Invariant. Using it, we can associate to a knot a series in the centre of the universal enveloping algebra of $L$, as long as we specify an $L$-invariant, non-degenerate, symmetric bilinear form on $L$. These series only depend on the knot isotopy class. Such a bilinear form can be chosen so that the construction of central elements agrees with the algebraic structure of the Quantum Lorentz Group. The algebraic properties of this kind of knot invariants will be another main topic of this article. 
We are mainly interested in the definition of numerical, rather than perturbative, knot invariants from infinite-dimensional representations of the Quantum Lorentz Group. We expect our expansions to relate to them, if we can define any, as their perturbation series at the origin. These issues will be dealt with in a separate work, $[\mathrm{FM}]$, where the convergence properties of the power series obtained are analysed. A major result therein is that even though the power series can have a zero radius of convergence, they are, at least in some cases, Borel-Gevrey summable. This indicates that some precise numerical knot invariants may be defined.

I finish by referring to the main motivation of this work, namely its possible applications to Quantum Gravity. For an example of the use of the unitary representations of the Lorentz Group in the construction of spin foam models for Quantum General Relativity we refer to [BC]. See also [NR] for its quantised counterpart.

\section{Contents}

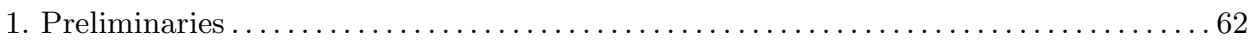

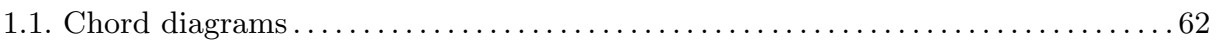

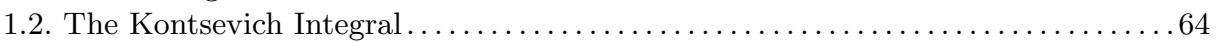

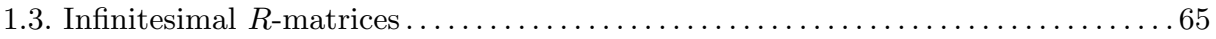

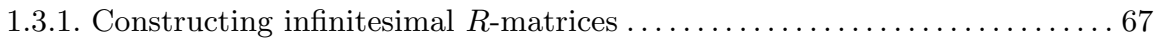

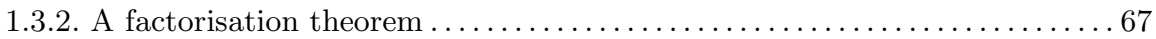

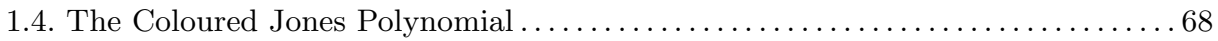

1.4.1. A representation interpretation of the $z$-Coloured Jones Polynomial . . . 70

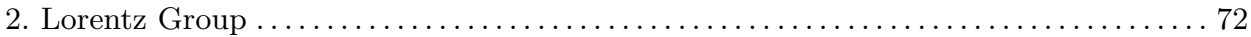

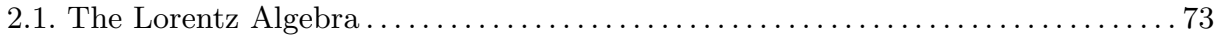

2.1.1. The irreducible balanced representations of the Lorentz Group . . . . . 76

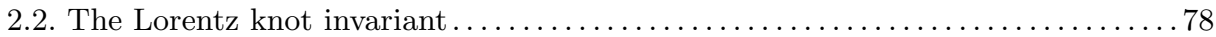

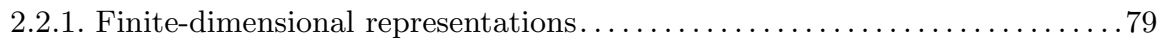

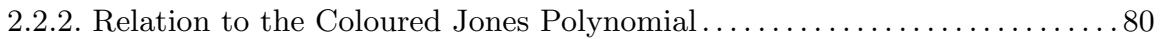

3. Relations to the approach of Buffenoir and Roche.................... 82

3.1. Representations of the Quantum Lorentz Group and $R$-matrices .........882

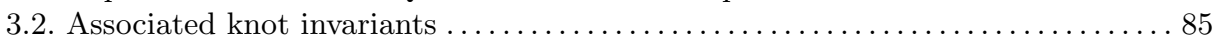

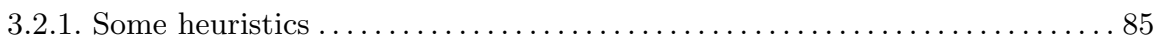

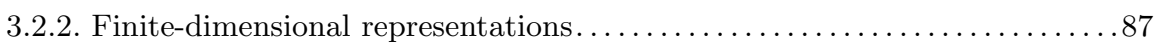

3.2.3. The series are convergent $h$-adically $\ldots \ldots \ldots \ldots \ldots \ldots \ldots \ldots \ldots \ldots \ldots \ldots$

3.2.4. The series define a $\mathbb{C}[[h]]$-valued knot invariant $\ldots \ldots \ldots \ldots \ldots \ldots \ldots \ldots \ldots 1$

\section{PRELIMINARIES}

1.1. Chord diagrams. We recall the definition of the algebra of chord diagrams, which is the target space for the Kontsevich Universal Knot Invariant. For more details see for example $[\mathrm{B}]$ or $[\mathrm{K}]$. A chord diagram is a finite set $w=\left\{c_{1}, \ldots, c_{n}\right\}$ of cardinality two, non-intersecting subsets of the 
oriented circle, modulo orientation preserving homeomorphisms. The subsets $c_{k}$ are called chords. We usually specify a chord diagram by drawing it as in Figure 1. In all the pictures we assume the circle oriented counterclockwise.

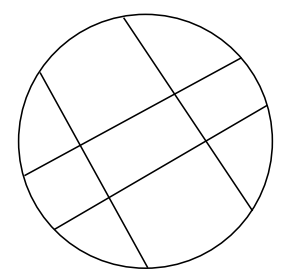

Fig. 1. A chord diagram with four chords

For each $n \geq 2$, let $V_{n}$ be the free $\mathbb{C}$-vector space on the set of all chord diagrams with $n$ chords, that is, the set of formal finite linear combinations $w=\sum_{i} \lambda_{i} w_{i}$, where $\lambda_{i} \in \mathbb{C}$ and $w_{i}$ is a chord diagram with $i$ chords for any $i$. Consider the vector subspace $4 T_{n}$ of $V_{n}$ generated by all linear combinations of chord diagrams of the form displayed in Figure 2. The three intervals considered in the circle can appear in an arbitrary order in $S^{1}$. For each $n \in \mathbb{N}_{0}=\{0,1,2, \ldots\}$, define the vector space $\mathcal{A}_{n}=V_{n} / 4 T_{n}$. We assume $\mathcal{A}_{0}=V_{0}$ and $\mathcal{A}_{1}=V_{1}$.

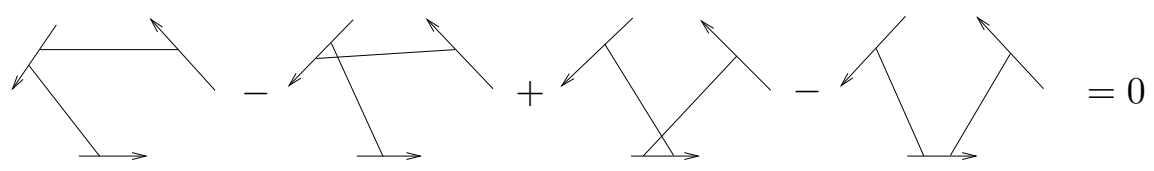

Fig. 2. 4-term relations

For any pair $m, n \in \mathbb{N}_{0}$, there exists a bilinear map \# : $\mathcal{A}_{n} \otimes \mathcal{A}_{m} \rightarrow$ $\mathcal{A}_{m+n}$, called the connected sum product. As its name says, it is performed by taking the connected sum of chord diagrams as in Figure 3.

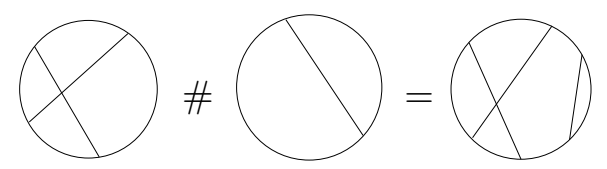

Fig. 3. Connected sum product

Obviously the product is not well defined in $V_{m} \otimes V_{n}$ since it depends on the points at which we break the circles. The connected sum makes sense only in $\mathcal{A}_{m} \otimes \mathcal{A}_{n}$, since we are considering the 4 -term relations. It is associative, commutative and it has a unit: the chord diagram without any chord. For more details see [B]. 
The vector space $\mathcal{A}_{m} \otimes \mathcal{A}_{m}$ is mapped via the connected sum product to $\mathcal{A}_{m+n}$. Therefore the direct sum $\mathcal{A}_{\text {fin }}=\bigoplus_{n \in \mathbb{N}_{0}} \mathcal{A}_{n}$ has a commutative and associative graded algebra structure. This permits us to conclude that the vector space

$$
\mathcal{A}=\prod_{n \in \mathbb{N}_{0}} \mathcal{A}_{n}
$$

has a structure of abelian algebra over the field of complex numbers. Call it the algebra of chord diagrams. The algebra $\mathcal{A}$ is the target space for the Kontsevich Universal Knot Invariant.

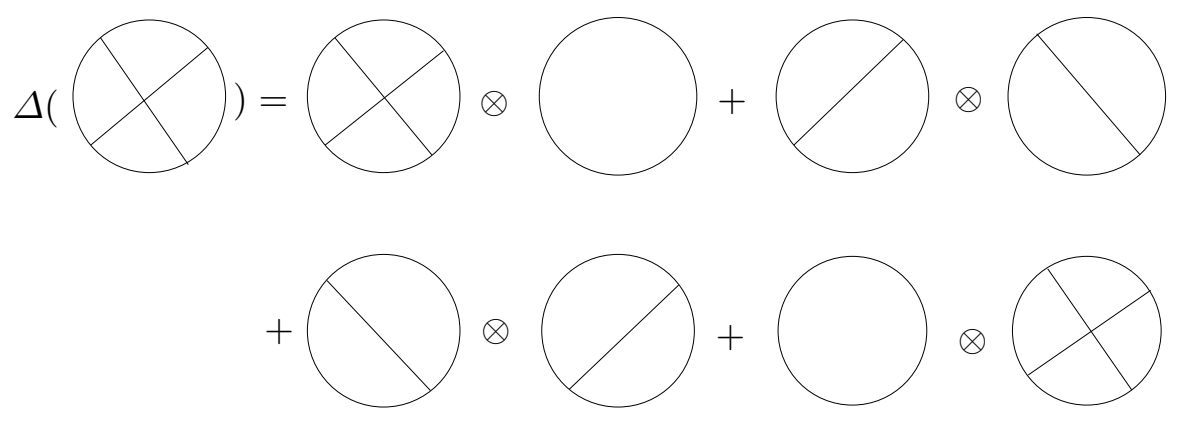

Fig.4. Coproduct maps

There also exist coproduct maps $\Delta: \mathcal{A}_{m} \rightarrow \bigoplus_{k+l=m} A_{k} \otimes \mathcal{A}_{l}$ which have the form of Figure 4 on chord diagrams. They extend to a linear map $\Delta: \mathcal{A} \rightarrow \mathcal{A} \widehat{\otimes} \mathcal{A}$. Here $\mathcal{A} \widehat{\otimes} \mathcal{A}$ is the vector space

$$
\prod_{m \in \mathbb{N}_{0}} \bigoplus_{k+l=m} \mathcal{A}_{k} \otimes \mathcal{A}_{l}
$$

Notice that $\mathcal{A} \otimes \mathcal{A}$ is a proper vector subspace of $\mathcal{A} \widehat{\otimes} \mathcal{A}$.

An element $w \in \mathcal{A}$ is called group-like if $\Delta(w)=w \widehat{\otimes} w$, that is, if $w=\sum_{n \in \mathbb{N}_{0}} w_{n}$ with $n \in \mathcal{A}_{n}$ for $n \in \mathbb{N}_{0}$ then

$$
\Delta\left(w_{n}\right)=\sum_{l+k=n} w_{k} \otimes w_{l}
$$

For example, $\exp (\ominus)$ is a group-like element. Here $\ominus$ is the unique chord diagram with only one chord. This is a trivial consequence of the fact that $\Delta(\ominus)=\ominus \otimes 1+1 \otimes \ominus$. Here 1 stands for the chord diagram without chords.

1.2. The Kontsevich Integral. We skip the definition of the (framed) Kontsevich Integral $\mathcal{Z}$, for which we refer for example to $[\mathrm{K}],[\mathrm{LM}]$ or $[\mathrm{W}]$. See also [B, CS] for the definition of the unframed version of the so-called Kontsevich Universal Knot Invariant. We take the normalisation of the Kontsevich Integral for which the value of the unknot is the wheels element $\Omega$ of [BLT]. 
That is, $\mathcal{Z}(O)=\mathbf{Z}(\infty)$ (cf. [B, p. 447]. This is a different normalisation from the one used in $[\mathrm{B}]$. We now gather the properties of the Kontsevich integral which we are going to use in what follows:

THEOREM 1. There exists an (oriented and framed) knot invariant $K \mapsto$ $\mathcal{Z}(K)$, where $\mathcal{Z}(K)$ is in the algebra $\mathcal{A}$ of chord diagrams. Given a framed knot $K, \mathcal{Z}(K)$ satisfies:

(i) $\mathcal{Z}(K)$ is group-like (cf. [B]).

(ii) If $K^{f}$ is obtained from $\mathcal{K}$ by changing its framing by a factor of 1 then $\mathcal{Z}\left(K^{f}\right)=\mathcal{Z}(K) \# \exp (\ominus)$ (cf. $\left.[\mathrm{LM}]\right)$.

(iii) If $K^{*}$ is the mirror image of $K$, and $\mathcal{Z}(K)=\sum_{n \in \mathbb{N}_{0}} w_{n}$ with $w_{n} \in \mathcal{A}_{n}$ for $n \in \mathbb{N}_{0}$ then $\mathcal{Z}\left(K^{*}\right)=\sum_{n \in \mathbb{N}_{0}}(-1)^{n} w_{n}$ (cf. [CS]).

(iv) If $K^{-}$is the knot obtained from $K$ by reversing the orientation of it then $\mathcal{Z}\left(K^{-}\right)=\sum_{n \in \mathbb{N}_{0}} S\left(w_{n}\right)$. Here $S: \mathcal{A}_{n} \rightarrow \mathcal{A}_{n}$ is the map that reverses the orientation of each chord diagram (cf. [CS]).

Suppose we are given a family of linear maps (weights) $W_{n}: \mathcal{A}_{n} \rightarrow \mathbb{C}$, $n \in \mathbb{N}_{0}$. A knot invariant whose value on each knot is a formal power series with coefficients in $\mathbb{C}$ is called canonical if it has the form

$$
K \mapsto \sum_{n \in \mathbb{N}_{0}} W_{n}\left(w_{n}\right) h^{n}
$$

whenever $\mathcal{Z}(K)=\sum_{n \in \mathbb{N}_{0}} w_{n}$ with $w_{n} \in \mathcal{A}_{n}$ for $n \in \mathbb{N}_{0}$.

1.3. Infinitesimal $R$-matrices. Let $\mathfrak{g}$ be a Lie algebra over the field $\mathbb{C}$. An infinitesimal R-matrix of $\mathfrak{g}$ is a symmetric tensor $t \in \mathfrak{g} \otimes \mathfrak{g}$ such that $[\Delta(X), t]=0$ for all $X \in \mathfrak{g}$. The commutator is taken in $U(\mathfrak{g}) \otimes U(\mathfrak{g})$, where $U(\mathfrak{g})$ denotes the universal enveloping algebra of $\mathfrak{g}$. The map $\Delta$ : $U(\mathfrak{g}) \rightarrow U(\mathfrak{g}) \otimes U(\mathfrak{g})$ is the standard coproduct in $U(\mathfrak{g})$. It satisfies $\Delta(X)=$ $X \otimes 1+1 \otimes X$ if $X \in \mathfrak{g}$.

Suppose we are given an infinitesimal $R$-matrix $t$. Write $t=\sum_{i} a_{i} \otimes b_{i}$. We will then have

$$
\sum_{i, j}\left(a_{j} a_{i} \otimes b_{i} \otimes b_{j}-a_{i} a_{j} \otimes b_{i} \otimes b_{j}+a_{i} \otimes a_{j} b_{i} \otimes b_{j}-a_{i} \otimes b_{i} a_{j} \otimes b_{j}\right)=0,
$$

which resembles the $4 T$ relations considered previously. Given a chord diagram $w$ and an infinitesimal $R$-matrix $t=\sum_{i} a_{i} \otimes b_{i}$ it is thus natural to construct an element $\phi_{t}(w)$ of $U(\mathfrak{g})$ in the following fashion: Start at an arbitrary point of the circle and go around it in the direction of its orientation. Order the chords of $w$ by the order in which you pass them as in Figure 5. Each chord has thus an initial point and an end point. Then go around the circle again and write (from right to left) $a_{i_{k}}$ or $b_{i_{k}}$ depending on whether you got to the initial or final point of the $k$ th chord. Finally, sum over all 


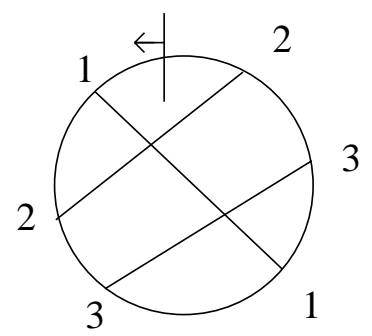

Fig. 5. Enumerating the chords of a chord diagram

the $i_{k}$ 's. For example for the chord diagram of Figure 5 the element $\phi_{t}(w)$ is

$$
\sum_{i_{1}, i_{2}, i_{3}} b_{i_{2}} b_{i_{3}} b_{i_{1}} a_{i_{3}} a_{i_{2}} a_{i_{1}} .
$$

See $[\mathrm{K}]$ or $[\mathrm{CV}]$ for more details. It is possible to prove that $\phi_{t}(w)$ is well defined as an element of $U(\mathfrak{g})$, that is, it does not depend on the starting point on the circle. Moreover:

THEOREM 2. Let $\mathfrak{g}$ be a Lie algebra and $t \in \mathfrak{g} \otimes \mathfrak{g}$ be an infinitesimal $R$ matrix. The linear map $\phi_{t}: V_{n} \rightarrow U(\mathfrak{g})$ satisfies the $4 T$ relations, therefore it descends to a linear map $\phi_{t}: \mathcal{A}_{n} \rightarrow U(\mathfrak{g})$. Moreover:

(i) The image of $\phi_{t}$ is contained in $\mathcal{C}(U(\mathfrak{g}))$, the centre of $U(\mathfrak{g})$.

(ii) The degree of $\phi_{t}(w)$ in $U(\mathfrak{g})$ with respect to the natural filtration of $U(\mathfrak{g})$ is not greater than twice the number of chords of $w$.

(iii) Given $w \in \mathcal{A}_{m}$ and $w^{\prime} \in \mathcal{A}_{n}$ we have $\phi_{t}\left(w \# w^{\prime}\right)=\phi_{t}(w) \phi_{t}\left(w^{\prime}\right)$.

(iv) Consider the map $\phi_{t, h}: \mathcal{A} \rightarrow \mathcal{C}(U(\mathfrak{g}))[[h]]$ such that if $w=\sum_{n \in \mathbb{N}_{0}} w_{n}$ with $w_{n} \in \mathcal{A}_{n}$ for each $n \in \mathbb{N}_{0}$ then

$$
\phi_{t, h}=\sum_{n \in \mathbb{N}_{0}} \phi_{t}\left(w_{n}\right) h^{n} .
$$

Then $\phi_{t, h}$ is a $\mathbb{C}$-algebra morphism.

Recall that the Kontsevich Integral is a sum of the form $\mathcal{Z}(K)=\sum_{n \in \mathbb{N}_{0}} w_{n}$ with $w_{n} \in \mathcal{A}_{n}$ for all $n \in \mathbb{N}_{0}$. Therefore, given an infinitesimal $R$-matrix $t$ in a Lie algebra $\mathfrak{g}$, we can define a knot invariant $\mathcal{Z}_{t}$ by

$$
\mathcal{Z}_{t}(K)=\phi_{t, h}(\mathcal{Z}(K))=\sum_{n} \phi_{t}\left(w_{n}\right) h^{n} .
$$

The target space of $\mathcal{Z}_{t}$ is therefore the $\mathbb{C}$-algebra of formal power series over the centre of $U(\mathfrak{g})$.

Suppose we are given a morphism $f: \mathcal{C}(U(\mathfrak{g})) \rightarrow \mathbb{C}$. Then composing it with $\mathcal{Z}(K)$ we obtain a canonical knot invariant $f \circ \mathcal{Z}_{t}$, that is:

$$
\left(f \circ \mathcal{Z}_{t}\right)(K)=\sum_{n} f\left(\phi_{t}\left(w_{n}\right)\right) h^{n} .
$$


It is not difficult to examine when this kind of knot invariants are unframed. Let $t=\sum_{i} a_{i} \otimes b_{i}$ be an infinitesimal $R$-matrix in a Lie algebra. Define $C_{t}=\sum_{i} a_{i} b_{i}=-\phi_{t}(\ominus)$. It is a central element of the universal enveloping algebra of $\mathfrak{g}$. Call it the quadratic central element associated with $t$. The infinitesimal $R$-matrix $t$ can be recovered from $C_{t}$ by the formula

$$
t=\frac{\Delta\left(C_{t}\right)-1 \otimes C_{t}-C_{t} \otimes 1}{2} .
$$

A morphism $f: \mathcal{C}(U(\mathfrak{g})) \rightarrow \mathbb{C}$ is said to be $t$-unframed if $f\left(C_{t}\right)=0$. From Theorems 1(ii) and 2(iii) it is straightforward to conclude that:

TheOREM 3. Let $\mathfrak{g}$ be a Lie algebra with an infinitesimal R-matrix $t$. Consider also a morphism $f$ from the centre of $U(\mathfrak{g})$ to $\mathbb{C}$. Then the knot invariant $f \circ \mathcal{Z}_{t}$ is unframed if and only if the morphism $f$ is t-unframed.

Notice that the Kontsevich integral of each knot is invertible in $\mathcal{A}$. This is because the term $w_{0} \in \mathcal{A}_{0}$ is the unit of $\mathcal{A}$.

1.3.1. Constructing infinitesimal $R$-matrices. There exists a standard way to construct infinitesimal $R$-matrices in a Lie algebra $\mathfrak{g}$. Suppose we are given a $\mathfrak{g}$-invariant, non-degenerate, symmetric bilinear form $\langle$,$\rangle in \mathfrak{g}$. Here $\mathfrak{g}$-invariance means that $\langle[X, Y], Z\rangle+\langle Y,[X, Z]\rangle=0$ for all $X, Y, Z \in \mathfrak{g}$. If $\mathfrak{g}$ is semisimple, the Cartan-Killing form has all these properties. Take a basis $\left\{X_{i}\right\}$ of $\mathfrak{g}$ and let $\left\{X^{i}\right\}$ be the dual basis of $\mathfrak{g}^{*}$. Then it is easy to show that for any $\lambda \in \mathbb{C}$ the tensor $t=\lambda \sum_{i} X_{i} \otimes X^{i}$ is an infinitesimal $R$-matrix of $\mathfrak{g}$. We are identifying $\mathfrak{g}^{*}$ with $\mathfrak{g}$ using the non-degenerate bilinear form $\langle$,$\rangle .$

Suppose $\mathfrak{g}$ is a semisimple Lie algebra and let $t=\sum_{i} a_{i} \otimes b_{i}$ be an infinitesimal $R$-matrix in $\mathfrak{g}$. Let also $\langle$,$\rangle denote the Cartan-Killing form$ on $\mathfrak{g}$. Then the map $\mathfrak{g} \rightarrow \mathfrak{g}$ such that $X \mapsto \sum_{i}\left\langle X, a_{i}\right\rangle b_{i}$ is an intertwiner of $\mathfrak{g}$ with respect to its adjoint representation. Therefore if $\mathfrak{g}$ is simple it is a $\lambda$-multiple of the identity. This permits us to conclude that $t=\lambda X_{i} \otimes X^{i}$.

Let us now look at the case of $\mathfrak{g}$ semisimple. Then $\mathfrak{g}$ has a unique decomposition $\mathfrak{g} \cong \mathfrak{g}_{1} \oplus \cdots \oplus \mathfrak{g}_{n}$, where each $\mathfrak{g}_{i}$ is a simple Lie algebra. The Cartan-Killing form in each $\mathfrak{g}_{i}$ will yield an infinitesimal $R$-matrix $t_{i}$ in each $\mathfrak{g}_{i}$. Obviously each linear combination $t=\lambda_{1} t_{1}+\cdots+\lambda_{n} t_{n}$ is an infinitesimal $R$-matrix for $\mathfrak{g}$. An argument similar to the one before proves that any infinitesimal $R$-matrix in $\mathfrak{g}$ is of the form above.

Note that if an infinitesimal $R$-matrix in a Lie algebra $\mathfrak{g}$ comes from a non-degenerate, symmetric and $\mathfrak{g}$-invariant bilinear form then our construction of central elements yields the same result as in [B] (cf. [CV]).

1.3.2. A factorisation theorem. Suppose the Lie algebra $\mathfrak{g} \cong \mathfrak{g}_{1} \oplus \mathfrak{g}_{2}$ is the direct sum of two Lie algebras. If $t_{1}$ and $t_{2}$ are infinitesimal $R$-matrices in $\mathfrak{g}_{1}$ and $\mathfrak{g}_{2}$ then $t=t_{1}+t_{2}$ is also an infinitesimal $R$-matrix in $\mathfrak{g}$. It is easy to prove that given a chord diagram $w$ we have the following identity (cf. 
$[\mathrm{B}])$ :

$$
\phi_{t}(w)=\left(\phi_{t_{1}} \otimes \phi_{t_{2}}\right) \Delta(w) .
$$

We are obviously considering the standard isomorphism $U(\mathfrak{g}) \cong U\left(\mathfrak{g}_{1}\right) \otimes$ $U\left(\mathfrak{g}_{2}\right)$ such that $(X, Y) \mapsto X \otimes 1+1 \otimes Y$ for $(X, Y) \in \mathfrak{g}$.

If we are given two algebra morphisms $f_{i}: \mathcal{C}\left(U\left(\mathfrak{g}_{i}\right)\right) \rightarrow \mathbb{C}, i=1,2$, then $f=f_{1} \otimes f_{1}$ is an algebra morphism $\mathcal{C}(U(\mathfrak{g})) \cong \mathcal{C}\left(U\left(\mathfrak{g}_{i}\right)\right) \otimes \mathcal{C}\left(U\left(\mathfrak{g}_{i}\right) \rightarrow \mathbb{C}\right.$. It thus makes sense to consider the knot invariant $f \circ \mathcal{Z}_{t}$. It can be expressed in a simple form in terms of $f_{i} \circ \mathcal{Z}_{t_{i}}, i=1,2$. In fact (see [BG]):

TheOREM 4. Given any (oriented and framed) knot $K$ we have

$$
\left(f \circ \mathcal{Z}_{t}\right)(K)=\left(f_{1} \circ \mathcal{Z}_{t_{1}}\right)(K) \times\left(f_{2} \circ \mathcal{Z}_{t_{2}}\right)(K)
$$

as formal power series.

Proof (taken from $[\mathrm{BG}]$ ). Write $\mathcal{Z}(K)=\sum_{n \in \mathbb{N}_{0}} w_{n}$ with $w_{n} \in \mathcal{A}_{n}$ for all $n \in \mathbb{N}_{0}$. We have

$$
\begin{aligned}
\left(f \circ \mathcal{Z}_{t}\right)(K) & =\sum_{n \in \mathbb{N}_{0}}\left(f \circ \phi_{t}\right)\left(w_{n}\right) h^{n}=\sum_{n \in \mathbb{N}_{0}}\left(f_{1} \otimes f_{2}\right) \circ\left(\phi_{t_{1}} \otimes \phi_{t_{2}}\right)\left(\Delta\left(w_{n}\right)\right) h^{n} \\
& =\sum_{n \in \mathbb{N}_{0}} \sum_{k+l=n}\left(f_{1} \otimes f_{2}\right) \circ\left(\phi_{t_{1}} \otimes \phi_{t_{2}}\right)\left(w_{k} \otimes w_{l}\right) h^{n} \\
& =\sum_{n \in \mathbb{N}_{0}} \sum_{k+l=n}\left[\left(f_{1} \circ \phi_{t_{1}}\right)\left(w_{k}\right)\right]\left[\left(f_{2} \circ \phi_{t_{2}}\right)\left(w_{l}\right)\right] h^{k+l} \\
& =\left(f_{1} \circ \mathcal{Z}_{t_{1}}\right)(K) \times\left(f_{2} \circ \mathcal{Z}_{t_{2}}\right)(K) .
\end{aligned}
$$

1.4. The Coloured Jones Polynomial. Let $\mathfrak{g}$ be a semisimple Lie algebra over $\mathbb{C}$. It is a well known result (see for example $[\mathrm{V}]$ ) that any algebra morphism $\mathcal{C}(U(\mathfrak{g})) \rightarrow \mathbb{C}$ is the central character of some representation of $\mathfrak{g}$, which can be infinite-dimensional. Recall that $\mathcal{C}(U(\mathfrak{g}))$ stands for the centre of $U(\mathfrak{g})$. To be more precise, let $\mathfrak{g}$ be any Lie algebra and $\varrho$ a representation of $\mathfrak{g}$ in the vector space $V$. Then $\varrho$ is said to admit a central character if every element of $\mathcal{C}(U(\mathfrak{g}))$ acts on $V$ as a multiple of the identity. In this case there exists an algebra morphism $\lambda_{\varrho}: \mathcal{C}(U(\mathfrak{g})) \rightarrow \mathbb{C}$ such that $\varrho(a)(v)=\lambda_{\varrho}(a) v$ for all $a \in \mathcal{C}(U(\mathfrak{g}))$ and $v \in V$. The algebra morphism $\lambda_{\varrho}$ is called the central character of the representation $\varrho$. In particular, if $\mathfrak{g}$ is a Lie algebra with an infinitesimal $R$-matrix $t$ then given any representation $\varrho$ of $\mathfrak{g}$ with a central character, we can construct the knot invariant $\lambda_{\varrho} \circ \mathcal{Z}_{t}$.

The Coloured Jones Polynomial is, up to normalisation, a particular example of this construction. Let $t$ be the infinitesimal $R$-matrix of $\mathfrak{s l}(2, \mathbb{C})$ corresponding to the bilinear form on it which is minus the Cartan-Killing form. Consider for any $\alpha \in \frac{1}{2} \mathbb{N}_{0}$ the representation $\varrho$ of $\mathfrak{s l}(2, \mathbb{C})$ with $\operatorname{spin} \alpha$; then $\stackrel{\alpha}{\varrho}$ admits a central character which we denote by $\lambda_{\alpha}$. Given a framed knot $K$ let $J^{\alpha}(K)$ denote the framed Coloured Jones Function of $K$. Notice 
that we "colour" the Jones polynomial with its spin of the representation, rather than with its dimension. The latter is the usual convention. We have

$$
\frac{J^{\alpha}(K)}{2 \alpha+1}=\left(\lambda_{\alpha} \circ \mathcal{Z}_{t}\right)(K), \quad \forall \alpha \in \frac{1}{2} \mathbb{N}_{0} .
$$

Write

$$
\frac{J^{\alpha}(K)}{2 \alpha+1}=\sum_{n \in \mathbb{N}_{0}} J_{n}^{\alpha}(K) h^{n} .
$$

It is known that $J_{n}^{a}(K)$ is a polynomial in $\alpha$ of degree at most $2 n$ (cf. $[\mathrm{MM}],[\mathrm{C}])$. This is a consequence of the fact that the centre of $U(\mathfrak{s l}(2, \mathbb{C}))$ is generated by the Casimir element of it, together with Theorem 2(ii). Therefore we can write

$$
\frac{J^{\alpha}(K)}{2 \alpha+1}=\sum_{n \in \mathbb{N}_{0}} \sum_{k=0}^{2 n} a_{k}^{(n)}(K) \alpha^{k} h^{n} .
$$

For any complex number $z$ it thus makes sense to consider the $z$-Coloured Jones Function, that is,

$$
\frac{J^{z}(K)}{2 z+1}=\sum_{n \in \mathbb{N}_{0}} P^{n}(K)(z) h^{n} .
$$

This yields a knot invariant whose value at a knot is a formal power series in two variables:

$$
K \mapsto \sum_{m, n \in \mathbb{N}_{0}} a_{k}^{(n)}(K) z^{k} h^{n}
$$

with $a_{k}^{(n)}(K)=0$ for $k>2 n$. It is an interesting task to investigate whether or not this kind of series defines an analytic function in two variables. As mentioned in the introduction, they have in general a zero radius of convergence, so this can only be made precise by taking a perturbation theory point of view (cf. $[\mathrm{FM}]$ ). This relates to the question of whether it is possible to define numerical knot invariants from infinite-dimensional representations of the Lorentz Group. Notice that if $\alpha$ is a half integer then

$$
\frac{J^{\alpha}(K)}{2 \alpha+1}=\sum_{m \in \mathbb{N}_{0}}\left(\sum_{k=0}^{2 n} a_{k}^{(n)}(K) \alpha^{k}\right) h^{n}
$$

defines an analytic function of $h$.

For the unknot $O$ the series $J^{z}(O) /(2 z+1)$ has a non-zero radius of convergence at any point $z \in \mathbb{C}$. The proof is not very difficult as we can have an explicit expression for the series. Define, for each $z \in \mathbb{C}$, the meromorphic function

$$
F_{z}(h)=\frac{1}{2 z+1} \frac{\sinh ((2 z+1) h / 2)}{\sinh (h / 2)} .
$$


Thus for each $\alpha \in \frac{1}{2} \mathbb{N}_{0}$ we have

$$
F_{\alpha}(h)=\frac{J^{\alpha}(O)}{2 \alpha+1}=\sum_{n \in \mathbb{N}_{0}} J_{n}^{\alpha}(O) h^{n} .
$$

Consider the expansion

$$
F_{z}(h)=\sum_{n \in \mathbb{N}_{0}} c(z)_{n} h^{n} .
$$

It is not difficult to check that each $c(z)_{n}$ is a polynomial in $z$ for fixed $n$. Moreover $c(\alpha)_{n}=J_{n}^{\alpha}(O)$ for all $\alpha \in \frac{1}{2} \mathbb{N}_{0}$. This implies

$$
\frac{J^{z}(O)}{2 z+1}=F_{z}(h)
$$

as power series in $h$. In particular the power series for the unknot are convergent. This means it makes sense to speak about the quantum dimension of the representations of spin $z$, to be defined later. To be more precise we give a meaning to their quantum dimension divided by their dimension as vector spaces. But notice that the dimension of a representation of $\operatorname{spin} z$ with $z \notin \frac{1}{2} \mathbb{N}_{0}$ is infinite. For some more explicit examples see [FM].

1.4.1. A representation interpretation of the z-Coloured Jones Polynomial. We can give an interpretation of the $z$-Coloured Jones Polynomial in the framework of central characters. To this end, define the following elements of $\mathfrak{s l}(2, \mathbb{C})$ :

$$
H=\left(\begin{array}{cc}
1 & 0 \\
0 & -1
\end{array}\right), \quad E=\left(\begin{array}{ll}
0 & 1 \\
0 & 0
\end{array}\right), \quad F=\left(\begin{array}{ll}
0 & 0 \\
1 & 0
\end{array}\right) .
$$

Then the infinitesimal $R$-matrix which we are considering in $\mathfrak{s l}(2, \mathbb{C})$ takes the form

$$
t=-\frac{1}{4}\left(E \otimes F+F \otimes E+\frac{H \otimes H}{2}\right) .
$$

Notice that $t$ is defined from the inner product in $\mathfrak{s l}(2, \mathbb{C})$ which is minus the Cartan-Killing form. In particular, the Casimir element $C$ of $\mathfrak{s l}(2, \mathbb{C})$ is equal to $-C_{t}$, where $C_{t}$ is the quadratic central element associated with $t$. Recall Subsection 1.3.

Given a half integer $\alpha$, the representation space $\stackrel{\alpha}{V}$ of the representation of spin $\alpha$ has a basis of the form $\left\{v_{0}, \ldots, v_{2 \alpha}\right\}$. The action of the elements $E, F$ and $H$ of $\mathfrak{s l}(2, \mathbb{C})$ in $\stackrel{\alpha}{V}$ is:

$$
H v_{k}=(k-\alpha) v_{k}, \quad E v_{k}=(2 \alpha-k) v_{k+1}, \quad F v_{k}=k v_{k-1} .
$$

For an arbitrary complex number $z \notin \frac{1}{2} \mathbb{N}_{0}$, it also makes sense to speak of the representation $\stackrel{z}{\varrho}$ of $\operatorname{spin} z$. Consider $\stackrel{z}{V}$ as being the infinite-dimensional 
vector space which has the basis $\left\{v_{2 z}, v_{2 z-1}, v_{2 z-2}, \ldots\right\}$. Then the representation $\stackrel{z}{\varrho}$ of $\operatorname{spin} z$ can be defined by

$$
\begin{aligned}
H v_{k} & =(k-z) v_{k}, & k & =2 z, 2 z-1, \ldots, \\
E v_{k} & =(2 z-k) v_{k+1}, & & k=2 z, 2 z-1, \ldots, \\
F v_{k} & =k v_{k-1}, & k & =2 z, 2 z-1, \ldots .
\end{aligned}
$$

The representations of spin $z \notin \frac{1}{2} \mathbb{N}_{0}$ have a central character $\lambda_{z}$, since it is easily proved that each intertwiner $\stackrel{z}{V} \rightarrow \stackrel{z}{V}$ must be a multiple of the identity. But (see [V, 4.10.2]) they are the unique irreducible cyclic highest weight representations with maximal weight $z$, relative to the usual Borel decomposition of $\mathfrak{s l}(2, \mathbb{C})$. Consider, given $z \in \mathbb{C}$, the framed knot invariant $\lambda_{z} \circ \mathcal{Z}_{t}$. If $\alpha$ is a half integer, $\lambda_{\alpha}$ is the central character of the usual representation of spin $\alpha$. Given a framed knot $K$ the invariant has the form

$$
\left(\lambda_{z} \circ \mathcal{Z}_{t}\right)(K)=\sum_{n \in \mathbb{N}_{0}} R_{n}^{z}(K) h^{n}
$$

where, by definition,

$$
R_{n}^{z}(K)=\left(\lambda_{z} \circ \phi_{t}\right)\left(w_{n}\right)=\sum_{n \in \mathbb{N}_{0}} \lambda_{z}\left(\phi_{t}\left(w_{n}\right)\right) h^{n}
$$

for

$$
\mathcal{Z}(K)=\sum_{n \in \mathbb{N}_{0}} w_{n}, \quad w_{n} \in \mathcal{A}_{n}, \forall n \in \mathbb{N}_{0}
$$

Also

$$
\frac{J^{\alpha}(K)}{2 z+1}=\sum_{n \in \mathbb{N}_{0}} R_{n}^{\alpha}(K) h^{n}, \quad \forall \alpha \in \frac{1}{2} \mathbb{N} .
$$

Suppose $w$ is a chord diagram with $n$ chords. Let us have a look at the dependence of $\lambda_{z}\left(\phi_{t}(w)\right)$ on $z$. It is not difficult to check that it is a polynomial in this variable of degree at most $2 n$. This is a trivial consequence of the definition of the central element $\phi_{t}(w)$ as well as the kind of action of the terms appearing in the infinitesimal $R$-matrix $t$ on $\stackrel{z}{V}$ (see also [V] or [FM]). In particular if $K$ is a framed knot, $R_{n}^{z}(K)$ is a polynomial in $z$. Since we also have $R_{n}^{\alpha}(K)=J_{n}^{\alpha}(K)$ for all $\alpha \in \frac{1}{2} \mathbb{N}_{0}$, we can conclude that

$$
\frac{J^{z}(K)}{2 z+1}=\left(\lambda_{z} \circ \mathcal{Z}\right)(K)
$$

which gives us an equivalent definition of the $z$-Coloured Jones Polynomial.

The central characters of the representations of imaginary spin are actually the infinitesimal characters (cf. [Kir]) of the unitary representations of $S L(2, \mathbb{R})$ in the principal series (cf. [L]) with the same parameter. Notice however that their derived representation in $\mathfrak{s l}(2, \mathbb{R}) \otimes_{\mathbb{R}} \mathbb{C} \cong \mathfrak{s l}(2, \mathbb{C})$ is not 
any of the representations of imaginary spin just defined. This is the point of view considered in $[\mathrm{FM}]$.

\section{LORENTZ GROUP}

Let $\mathfrak{g}$ be a semisimple Lie algebra. As proved by Drinfeld in [D], there is a one-to-one correspondence between gauge equivalence classes of quantised universal enveloping algebras $\mathcal{H}$ of $\mathfrak{g}$ over $\mathbb{C}[[h]]$ (cf. $[\mathrm{K}]$ ) and infinitesimal $R$-matrices in $\mathfrak{g}$. Let us be more explicit about this. It is implicit in the definition of a quantised universal enveloping algebra $\mathcal{H}$ that there exists a $\mathbb{C}$-algebra morphism $f: \mathcal{H} / h \mathcal{H} \rightarrow U(\mathfrak{g})$. Having chosen such a morphism, the canonical 2-tensor of $\mathcal{A}$ is defined as $t=f\left(\left(R_{21} R-1\right) / h\right)$. It is an infinitesimal $R$-matrix of $\mathcal{A}$. Here $R$ denotes the universal $R$-matrix of $\mathcal{H}$. If $\mathcal{H}$ quantises the pair $(\mathfrak{g}, r)$ where $r$ is a classical $r$-matrix in $\mathfrak{g}$ (see $[\mathrm{CP}]$ ), then $t$ is the symmetrisation of $r$. Each quantised universal enveloping algebra can be given a structure of ribbon quasi Hopf algebra (cf. [AC]), and therefore there is a knot invariant attached to each finite-dimensional representation of it, or what is the same, of $\mathfrak{g}$. These knot invariants take their values in the ring of formal power series over $\mathbb{C}$. If the representation used is finitedimensional and irreducible then it has a central character. In particular, the framework of the last section can be applied, using for example the infinitesimal $R$-matrix $t$ which is the canonical 2-tensor of $\mathcal{H}$. It is a deep result that with these choices the two approaches for knot invariants are the same, up to division by the dimension of the representation considered. To be more precise we also need to change the sign of the infinitesimal $R$-matrix $t$ (cf. $[\mathrm{K}])$.

If we consider a $q$-deformation $\mathcal{A}$ of the universal enveloping algebra of a Lie algebra $\mathfrak{g}$, then no such classification of gauge equivalence classes of quantised universal enveloping algebras exists. But sometimes it is possible to give a meaning to the formula for $t$. This is because we have a $q$-parametrised family of braided Hopf algebras that tends to the universal enveloping algebra of $\mathfrak{g}$ as $q$ goes to 1 , or alternatively because $\mathcal{A}$ quantises the pair $(\mathfrak{g}, r)$ where $r$ is an $r$-matrix in $\mathfrak{g}$.

As mentioned in the introduction, despite the fact that the $q$-DrinfeldJimbo quantised universal enveloping algebras $U_{q}(\mathfrak{g})$ of semisimple Lie algebras are not ribbon Hopf algebras, their category of finite-dimensional representations is a ribbon category. That is, they have formal $R$-matrices and ribbon elements, which make sense when acting in their finite-dimensional representations. The target space for the knot invariants in this context is the complex plane. These numerical knot invariants can be obtained, up to rescaling, by summing the power series which appear in the context of $h$-adic Drinfeld-Jimbo algebras; in other words, by summing the power series that 
come from the approach making use of the Kontsevich Integral and of the infinitesimal $R$-matrix which is the heuristic canonical 2-tensor $t$ of $U_{q}(\mathfrak{g})$.

Let us now pass to the Quantum Lorentz Group $\mathcal{D}$ as defined in [BR1] and [BR2]. It is a quantum group depending on a parameter $q \in(0,1)$. As said in the introduction, we wish to analyse the question of whether or not there exists a knot theory attached to the infinite-dimensional representations of $\mathcal{D}$. The situation is more or less the same as in the case of $q$-Drinfeld-Jimbo algebras. Namely we have a heuristic $R$-matrix which comes from its structure of a quantum double as well as a heuristic ribbon element. It is possible to describe how they act in the unitary representations of $\mathcal{D}$. The situation is simpler if the minimal spin of the representation is zero, in which case the representation is said to be balanced. Representations of this kind are called simple in $[\mathrm{NR}]$. In this context, the ribbon element acts as the identity and therefore the knot invariants obtained will be unframed. These invariants express as infinite sums as we will see in Section 3.

One natural thing to do would be to analyse whether the "derivatives" of these sums define or not Vassiliev invariants, or whether it is possible to give them a meaning in the framework of the Kontsevich Universal Invariant. It is not difficult to find an expression for the heuristic canonical 2-tensor of the Quantum Lorentz Group. Also the unitary representations of the Quantum Lorentz Group in the principal and complementary series have a classical counterpart. They are infinite-dimensional representations of the Lie algebra of the Lorentz Group which admit a central character and therefore the framework of the last section can be used. This is the program we wish to undertake now.

2.1. The Lorentz Algebra. Consider the complex Lie group $S L(2, \mathbb{C})$. Its Lie algebra $\mathfrak{s l}(2, \mathbb{C})$ is a complex Lie algebra of dimension 3 . A basis of $\mathfrak{s l}(2, \mathbb{C})$ is $\left\{\sigma_{X}, \sigma_{Y}, \sigma_{Z}\right\}$ where

$$
\sigma_{X}=\frac{1}{2}\left(\begin{array}{cc}
i & 0 \\
0 & -i
\end{array}\right), \quad \sigma_{Y}=\frac{1}{2}\left(\begin{array}{cc}
0 & i \\
i & 0
\end{array}\right), \quad \sigma_{Z}=\frac{1}{2}\left(\begin{array}{cc}
0 & -1 \\
1 & 0
\end{array}\right) .
$$

The commutation relations are

$$
\left[\sigma_{X}, \sigma_{Y}\right]=\sigma_{Z}, \quad\left[\sigma_{Y}, \sigma_{Z}\right]=\sigma_{X}, \quad\left[\sigma_{Z}, \sigma_{X}\right]=\sigma_{Y}
$$

We can also consider a different basis $\left\{H_{+}, H_{-}, H_{3}\right\}$, where

$$
H_{+}=i \sigma_{X}-\sigma_{Y}, \quad H_{-}=i \sigma_{X}+\sigma_{Y}, \quad H_{3}=i \sigma_{Z},
$$

the new commutation relations being

$$
\left[H_{+}, H_{3}\right]=-H_{+}, \quad\left[H_{-}, H_{3}\right]=H_{-}, \quad\left[H_{+}, H_{-}\right]=2 H_{3} .
$$


Restricting the ground field we are working in to $\mathbb{R}$, we obtain a 6-dimensional real Lie algebra $\mathfrak{s l}(2, \mathbb{C})_{\mathbb{R}}$, the realification of $\mathfrak{s l}(2, \mathbb{C})$. It is isomorphic to the Lie algebra of the Lorentz Group.

Definition 5. The Lorentz Lie algebra $L$ is defined to be the complex Lie algebra which is the complexification of $\mathfrak{s l}(2, \mathbb{C})_{\mathbb{R}}$. That is, $L=$ $\mathfrak{s l}(2, \mathbb{C})_{\mathbb{R}} \otimes_{\mathbb{R}} \mathbb{C}$. It is therefore a complex Lie algebra of dimension 6 . The Lorentz Algebra is the complex algebra $U(L)$ which is the universal enveloping algebra of the complex Lie algebra $L$.

The set $\left\{\sigma_{X}, B_{X}=-i \sigma_{X}, \sigma_{Y}, B_{Y}=-i \sigma_{Y}, \sigma_{Z}, B_{Z}=-i \sigma_{Z}\right\}$ is a real basis of $\mathfrak{s l}(2, \mathbb{C})_{\mathbb{R}}$, and thus a complex basis of $L$. The commutation relations are

$$
\begin{aligned}
& {\left[\sigma_{X}, \sigma_{Y}\right]=\sigma_{Z}, \quad\left[\sigma_{Y}, \sigma_{Z}\right]=\sigma_{X}, \quad\left[\sigma_{Z}, \sigma_{X}\right]=\sigma_{Y},} \\
& {\left[\sigma_{Z}, B_{X}\right]=B_{Y}, \quad\left[\sigma_{Y}, B_{X}\right]=-B_{Z}, \quad\left[\sigma_{X}, B_{X}\right]=0,} \\
& {\left[\sigma_{Z}, B_{Y}\right]=-B_{X}, \quad\left[\sigma_{Y}, B_{Y}\right]=0, \quad\left[\sigma_{X}, B_{Y}\right]=B_{Z},} \\
& {\left[\sigma_{Z}, B_{Z}\right]=0, \quad\left[\sigma_{Y}, B_{Z}\right]=B_{X}, \quad\left[\sigma_{X}, B_{Z}\right]=-B_{Y},} \\
& {\left[B_{X}, B_{Y}\right]=-\sigma_{Z}, \quad\left[B_{Y}, B_{Z}\right]=-\sigma_{X}, \quad\left[B_{Z}, B_{X}\right]=-\sigma_{Y} .}
\end{aligned}
$$

We can also consider the basis $\left\{H_{+}, H_{-}, H_{3}, F_{+}, F_{-}, F_{3}\right\}$ of $L$, where

$$
\begin{aligned}
& H_{+}=i \sigma_{X}-\sigma_{Y}, \quad H_{-}=i \sigma_{X}+\sigma_{Y}, \quad H_{3}=i \sigma_{Z}, \\
& F_{+}=i B_{X}-B_{Y}, \quad F_{-}=i B_{X}+B_{Y}, \quad F_{3}=i B_{Z} .
\end{aligned}
$$

The new commutation relations are

$$
\begin{gathered}
{\left[H_{+}, H_{3}\right]=-H_{+}, \quad\left[H_{-}, H_{3}\right]=H_{-}, \quad\left[H_{+}, H_{-}\right]=2 H_{3},} \\
{\left[F_{+}, H_{+}\right]=\left[H_{-}, F_{-}\right]=\left[H_{3}, F_{3}\right]=0,} \\
{\left[H_{+}, F_{3}\right]=-F_{+},\left[H_{-}, F_{3}\right]=F_{-},} \\
{\left[H_{+}, F_{-}\right]=-\left[H_{-}, F_{+}\right]=2 F_{3},} \\
{\left[F_{+}, H_{3}\right]=-F_{+},\left[F_{-}, H_{3}\right]=F_{-},} \\
{\left[F_{+}, F_{3}\right]=H_{+}, \quad\left[F_{-}, F_{3}\right]=-H_{-}, \quad\left[F_{+}, F_{-}\right]=-2 H_{3} .}
\end{gathered}
$$

The following simple theorem will be one of the most important in our discussion.

THEOREM 6 . There exists a unique isomorphism of complex Lie algebras $\tau: \mathfrak{s l}(2, \mathbb{C}) \oplus \mathfrak{s l}(2, \mathbb{C}) \rightarrow L \cong \mathfrak{s l}(2, \mathbb{C})_{\mathbb{R}} \otimes_{\mathbb{R}} \mathbb{C}$ such that

$$
\begin{gathered}
\sigma_{X} \oplus 0 \mapsto \frac{\sigma_{X}-i \sigma_{X} \otimes i}{2}=\frac{\sigma_{X}+i B_{X}}{2}, \\
0 \oplus \sigma_{X} \mapsto \frac{\sigma_{X}+i \sigma_{X} \otimes i}{2}=\frac{\sigma_{X}-i B_{X}}{2},
\end{gathered}
$$




$$
\begin{gathered}
\sigma_{Y} \oplus 0 \mapsto \frac{\sigma_{Y}-i \sigma_{Y} \otimes i}{2}=\frac{\sigma_{Y}+i B_{Y}}{2}, \\
0 \oplus \sigma_{Y} \mapsto \frac{\sigma_{Y}+i \sigma_{Y} \otimes i}{2}=\frac{\sigma_{Y}-i B_{Y}}{2}, \\
\sigma_{Z} \oplus 0 \mapsto \frac{\sigma_{Z}-i \sigma_{Z} \otimes i}{2}=\frac{\sigma_{Z}+i B_{Z}}{2}, \\
0 \oplus \sigma_{Z} \mapsto \frac{\sigma_{Z}+i \sigma_{Z} \otimes i}{2}=\frac{\sigma_{Z}-i B_{Z}}{2} .
\end{gathered}
$$

Thus we also have a Hopf algebra isomorphism

$$
\tau: U(\mathfrak{s l}(2, \mathbb{C})) \otimes U(\mathfrak{s l}(2, \mathbb{C})) \rightarrow U(L)
$$

Proof. Easy calculations.

Given $X \in \mathfrak{s l}(2, \mathbb{C})$, define $X^{l}=\tau(X \oplus 0), X^{r}=\tau(0 \oplus X)$ and analogously for $X \in U(\mathfrak{s l}(2, \mathbb{C}))$. We have

$$
\begin{aligned}
& H_{+}^{l}=\frac{H_{+}+i F_{+}}{2}, \quad H_{-}^{l}=\frac{H_{-}+i F_{-}}{2}, \quad H_{3}^{l}=\frac{H_{3}+i F_{3}}{2}, \\
& H_{+}^{r}=\frac{H_{+}-i F_{+}}{2}, \quad H_{-}^{r}=\frac{H_{-}-i F_{-}}{2}, \quad H_{3}^{r}=\frac{H_{3}-i F_{3}}{2} .
\end{aligned}
$$

Consider also $C^{l}=\tau(C \otimes 1)$ and $C^{r}=\tau(1 \otimes C)$, where $C$ is the Casimir element of $\mathfrak{s l}(2, \mathbb{C})$ defined in 1.4. The elements $C^{l}$ and $C^{r}$ are called the left and right Casimirs and their explicit expressions are

$$
\begin{aligned}
4 C^{l}= & \frac{H_{3}^{2}-F_{3}^{2}}{2}+i \frac{H_{3} F_{3}}{2}+i \frac{F_{3} H_{3}}{2} \\
& +\frac{H_{+} H_{-}}{4}+i \frac{H_{+} F_{-}}{4}+i \frac{F_{+} H_{-}}{4}-\frac{F_{+} F_{-}}{4} \\
& +\frac{H_{-} H_{+}}{4}+i \frac{H_{-} F_{+}}{4}+i \frac{F_{-} H+}{4}-\frac{F_{-} F_{+}}{4}, \\
4 C^{r}= & \frac{H_{3}^{2}-F_{3}^{2}}{2}-i \frac{H_{3} F_{3}}{2}-i \frac{F_{3} H_{3}}{2} \\
& +\frac{H_{+} H_{-}}{4}-i \frac{H_{+} F_{-}}{4}-i \frac{F_{+} H_{-}}{4}-\frac{F_{+} F_{-}}{4} \\
& +\frac{H_{-} H_{+}}{4}-i \frac{H_{-} F_{+}}{4}-i \frac{F_{-} H+}{4}-\frac{F_{-} F_{+}}{4} .
\end{aligned}
$$

We can also consider the left and right image under $\tau \otimes \tau$ of the infinitesimal $R$-matrix of $U(\mathfrak{s l}(2, \mathbb{C}))$. We now take $t \in \mathfrak{s l}(2, \mathbb{C}) \otimes \mathfrak{s l}(2, \mathbb{C})$ to be the infinitesimal $R$-matrix coming from the Cartan-Killing form, that is, minus the one considered in 1.4. These left and right infinitesimal $R$-matrices are: 


$$
\begin{aligned}
4 t^{l}= & \frac{H_{3} \otimes H_{3}}{2}-\frac{F_{3} \otimes F_{3}}{2}+i \frac{H_{3} \otimes F_{3}}{2}+i \frac{F_{3} \otimes H_{3}}{2} \\
& +\frac{H_{+} \otimes H_{-}}{4}+i \frac{H_{+} \otimes F_{-}}{4}+i \frac{F_{+} \otimes H_{-}}{4}-\frac{F_{+} \otimes F_{-}}{4} \\
& +\frac{H_{-} \otimes H_{+}}{4}+i \frac{H_{-} \otimes F_{+}}{4}+i \frac{F_{-} \otimes H+}{4}-\frac{F_{-} \otimes F_{+}}{4}, \\
4 t^{r}= & \frac{H_{3} \otimes H_{3}}{2}-\frac{F_{3} \otimes F_{3}}{2}-i \frac{H_{3} \otimes F_{3}}{2}-i \frac{F_{3} \otimes H_{3}}{2} \\
& +\frac{H_{+} \otimes H_{-}}{4}-i \frac{H_{+} \otimes F_{-}}{4}-i \frac{F_{+} \otimes H_{-}}{4}-\frac{F_{+} \otimes F_{-}}{4} \\
& +\frac{H_{-} \otimes H_{+}}{4}-i \frac{H_{-} \otimes F_{+}}{4}-i \frac{F_{-} \otimes H+}{4}-\frac{F_{-} \otimes F_{+}}{4} .
\end{aligned}
$$

Any linear combination $a t^{l}+b t^{r}$ of the left and right infinitesimal $R$-matrices is an infinitesimal $R$-matrix for $L$. We wish to consider the combination $t_{L}=t^{l}-t^{r}$, that is,

$t_{L}=i \frac{1}{4} H_{3} \otimes F_{3}+\frac{1}{4} i F_{3} \otimes H_{3}+\frac{i}{8} H_{-} \otimes F_{+}+\frac{i}{8} F_{-} \otimes H_{+}+\frac{i}{8} H_{+} \otimes F_{-}+\frac{i}{8} F_{+} \otimes H_{-}$.

Notice another expression of it:

$t_{L}=\frac{i}{8}\left(B_{X} \otimes \sigma_{X}+\sigma_{X} \otimes B_{X}+B_{Y} \otimes \sigma_{Y}+\sigma_{Y} \otimes B_{Y}+B_{Z} \otimes \sigma_{Z}+\sigma_{Z} \otimes B_{Z}\right)$.

The quadratic central element of $U(L)$ associated with $t_{L}$ is

$$
C_{L}=C_{t_{L}}=i \frac{H_{3} F_{3}}{4}+i \frac{F_{3} H_{3}}{4}+i \frac{H_{+} F_{-}}{8}+i \frac{F_{+} H_{-}}{8}+i \frac{H_{-} F_{+}}{8}+i \frac{F_{-} H_{+}}{8} .
$$

The reason why we consider this particular combination of the left and right infinitesimal $R$-matrices is because it corresponds to the heuristic canonical 2-tensor of the Quantum Lorentz Group considered in [BR2]. Notice that it is the symmetrisation of the classical $r$-matrix of $\mathfrak{s l}(2, \mathbb{C})_{\mathbb{R}}$ (see $[\mathrm{BNR}$, p. 4969]). See also [FM]. We shall see later (Theorem 25) that it is the right one.

2.1.1. The irreducible balanced representations of the Lorentz Group. Given a complex number $p=|p| e^{i \theta}, 0 \leq \theta<2 \pi$, different from zero, we define once for all the square root $\sqrt{p}$ of $p$ to be $\sqrt{|p| e^{i \theta}}=\sqrt{|p|} e^{i \theta / 2}$. For $m \in \mathbb{Z}$ define the set $W_{m}=\left\{p \in \mathbb{C}:|p| \notin \mathbb{N}_{|m|+1}\right\}$, where, in general, $\mathbb{N}_{m}=\{m, m+1, \ldots\}$ for any $m \in \mathbb{N}$. Consider the set $\mathcal{P}=\{(m, p): m \in \mathbb{Z}$, $\left.p \in W_{m}\right\}$. Define, for any $\alpha \in \mathbb{N}$ and $(m, p) \in \mathcal{P}$,

$$
C_{\alpha}(m, p)=\frac{i}{\alpha} \sqrt{\frac{\left(\alpha^{2}-p^{2}\right)\left(\alpha^{2}-m^{2}\right)}{4 \alpha^{2}-1}}, \quad B_{\alpha}(m, p)=\frac{i p m}{\alpha(\alpha+1)} .
$$

Thus $C_{\alpha}(m, p) \neq 0$ for $\alpha=|m|+1,|m|+2, \ldots$ and all $p \in W_{m}$. 
Consider the complex vector space

$$
V(m)=\bigoplus_{\alpha \in \mathbb{N}_{|m|}} \stackrel{\alpha}{V}
$$

where $\stackrel{\alpha}{V}$ denotes the representation space of the representation of $\mathfrak{s l}(2, \mathbb{C})$ of spin $\alpha$. The set $\left\{\stackrel{\alpha}{v}_{i}: i=-\alpha,-\alpha+1, \ldots, \alpha ; \alpha \in \mathbb{N}_{|m|}\right\}$ is a basis of $V(m)$. Consider the inner product in $V(m)$ for which this basis is orthonormal. Define also $\bar{V}(m)$ to be the Hilbert space which is the completion of $V(m)$.

Given $(m, p) \in \mathcal{P}$, consider the following linear operators acting on $V(m)$ :

$$
\begin{aligned}
& H_{3} \stackrel{\alpha}{v}_{k}=k \stackrel{\alpha}{v}{ }_{k}, \\
& H_{-} \stackrel{\alpha}{k}_{k}=\sqrt{(\alpha+k)(\alpha-k+1)}{ }^{\alpha}{ }_{k-1}, \\
& H_{+} \stackrel{\alpha}{v}_{k}=\sqrt{(\alpha+k+1)(\alpha-k)} \stackrel{\alpha}{v}_{k+1}, \\
& F_{+} \stackrel{\alpha}{v}_{k}=C_{\alpha}(m, p) \sqrt{(\alpha-k)(\alpha-k-1)}{ }^{\alpha-1}{ }_{k+1} \\
& \text { - } B_{\alpha}(m, p) \sqrt{(\alpha+k+1)(\alpha-k)} v_{k+1}^{\alpha} \\
& +C_{\alpha+1}(m, p) \sqrt{(\alpha+k+1)(\alpha+k+2)}{ }_{v}^{\alpha+1}{ }_{k+1} \text {, } \\
& F_{-} \stackrel{\alpha}{v}_{k}=-C_{\alpha}(m, p) \sqrt{(\alpha+k)(\alpha+k-1)}{ }^{\alpha-1}{ }_{k-1} \\
& -B_{\alpha}(m, p) \sqrt{(\alpha-k+1)(\alpha+k)} v_{k-1}^{\alpha} \\
& -C_{\alpha+1}(m, p) \sqrt{(\alpha+1)^{2}-k^{2}}{ }_{v}^{\alpha+1}{ }_{k-1}, \\
& F_{3} \stackrel{\alpha}{v}_{k}=C_{\alpha}(m, p) \sqrt{\alpha^{2}-k^{2}}{ }^{\alpha-1}{ }_{k}-B_{\alpha}(m, p) k v_{k} \\
& -C_{\alpha+1}(m, p) \sqrt{(\alpha+1)^{2}-k^{2}}{ }_{v}^{\alpha+1}{ }_{k} \text {, } \\
& k=-\alpha,-\alpha+1, \ldots, \alpha, \alpha \in \mathbb{N}_{|m|} .
\end{aligned}
$$

Obviously we are considering $\stackrel{\alpha}{v}_{k}=0$ if $k>\alpha$ or $k<-\alpha$. We have the following theorem, whose proof can be found in [GMS]:

Theorem 7. If $(m, p) \in \mathcal{P}$, the operators $H_{-}, H_{+}, H_{3}, F_{-}, F_{+}, F_{3}$ define an infinite-dimensional representation of the Lorentz Algebra.

Notice that the representations $(m, p)$ and $(-m,-p)$ are equivalent. This statement has a trivial proof.

Denote the representations above by $\{\varrho(m, p):(m, p) \in \mathcal{P}\}$. One can prove with no difficulty that they have a central character, for any intertwiner $V(m) \rightarrow V(m)$ needs to send each space $\stackrel{\alpha}{V}$ to itself and act on it as a multiple of the identity. Considering the action of $F_{+}$, for example, we conclude that the multiples are the same in each space $\stackrel{\alpha}{V}$. Therefore we have 
TheOREM 8. For any $(m, p) \in \mathcal{P}$ the representation $\varrho(m, p)$ of $L$ has a central character $\lambda_{m, p}$.

For any $(m, p) \in \mathcal{P}$ the representation $\varrho(m, p)$ of $L$ can always be integrated to a representation $R(m, p)$ of the Lorentz Group in the completion $\bar{V}(m)$ of $V(m)$, or to be more precise of its connected component of the identity. The representation is unitary if and only if $p$ is purely imaginary, for any $m \in \mathbb{N}_{0}$, in which case the representation is said to belong to the principal series, or if $m=0$ and $p \in[0,1)$, in which case the representation is said to belong to the complementary series. The vector space $V(m)$ is contained in the space of smooth vectors (cf. [Kir]) of $V(m)$; thus $\lambda_{m, p}$ is the infinitesimal character of $R(m, p)$, in the unitary case. This unifies the approach here with the approach in $[\mathrm{FM}]$.

The parameter $m$ is called the minimal spin of the representation. A representation is called balanced if its minimal spin is 0. Balanced representations depend therefore on a parameter $p \in W_{0}$. Denote them by $\left\{\varrho_{p}: p \in W_{0}\right\}$. Two balanced representations $\varrho_{p}$ and $\varrho_{q}$ of $L$ are equivalent if and only if $p=q$ or $p=-q$. These representations were used in [BC] for the construction of a spin foam model for Quantum Gravity. The extension of that work to their quantised counterpart was dealt with in $[\mathrm{NR}]$.

Since the representations $\{\varrho(m, p):(m, p) \in \mathcal{P}\}$ have a central character, the left and right Casimirs defined in 2.1 act on $V(m)$ as multiples of the identity. These multiples are the following functions of $m$ and $p$ : $\left(p^{2}+2 m p+m^{2}-1\right) / 8$ for $C^{l}$ and $\left(p^{2}-2 m p+m^{2}-1\right) / 8$ for $C^{r}$. Therefore we have

Proposition 9. If the infinitesimal R-matrix on $U(L)$ is the tensor $t_{L}$ defined in 2.1 , then the central characters $\left\{\lambda_{p}: p \in W_{0}\right\}$ of the balanced representations are $t_{L}$-unframed (recall the terminology introduced before Theorem 3$)$.

This can obviously be proved without using the explicit expression of the action of the Casimir elements.

Notice also that we can consider the minimal spin of the representations considered to be a half integer, making an obvious change in the form of the representation. This kind of representations cannot be integrated to representations of the Lorentz Group, even though they define representations of $S L(2, \mathbb{C})$. They are called two-valued representations of the Lorentz Group in [GMS].

2.2. The Lorentz knot invariant. Consider again the infinitesimal $R$-matrix $t_{L}=t^{l}-t^{r}$ of the Lorentz Lie Algebra. We consider for each $(m, p) \in \mathcal{P}$ the representation $\varrho(m, p)$ of $L$. It has a central character $\lambda_{m, p}$. We propose to consider the framed knot invariants $\{X(m, p):(m, p) \in \mathcal{P}\}$ 
such that for any knot:

$$
K \mapsto X(m, p, K)=\left(\lambda_{m, p} \circ \mathcal{Z}_{t}\right)(K) .
$$

Recall the notation of 1.3 . Notice that $X(m, p)=X(-m,-p)$, for the representations $\varrho_{m, p}$ and $\varrho_{-m,-p}$ are equivalent.

The value of $X(m, p)$ in a framed knot $K$ is therefore a formal power series with coefficients in $\mathbb{C}$. It is a difficult task to analyse the analytic properties of such power series. We expect they will be perturbation series for some numerical knot invariants that can be defined (cf. [FM]).

As we have seen, if $m=0$, that is, in the case of balanced representations, the central character $\lambda_{p}$ is $t_{L}$-unframed. This is also the case for $p=0$. Notice we have an explicit expression for the action of the left and right Casimir elements of $L$. This yields

TheOREM 10. The knot invariant $X(m, p)$ with $(m, p) \in \mathcal{P}$ is unframed if and only if $m=0$ or $p=0$.

Obviously, for different combinations of the left and right infinitesimal $R$-matrices, the representations which have unframed central characters with respect to the resulting infinitesimal $R$-matrix (see 1.3.1) are different. This gives us a way to define an unframed knot invariant from any $(m, p) \in \mathcal{P}$. But notice this can be done without changing the infinitesimal $R$-matrix $t_{L}$ of $L$, since we know how the invariants behave with respect to framing (cf. Theorem 1).

2.2.1. Finite-dimensional representations. Let us now analyse the knot invariants that come from finite-dimensional representations of the Lorentz Group. We are mainly interested in the representations which are irreducible.

Since we have the isomorphism $U(L) \cong U(\mathfrak{s l}(2, \mathbb{C})) \otimes U(\mathfrak{s l}(2, \mathbb{C}))$, the finite-dimensional irreducible representations of $U(L)$, or what is the same of $L$, are classified by pairs $(\alpha, \beta)$ of half integers. That is, each finitedimensional irreducible representation of $L$ is of the form $\stackrel{\alpha}{\varrho} \otimes \varrho^{\beta}$ as a representation of $U(L) \cong U(\mathfrak{s l}(2, \mathbb{C})) \otimes U(\mathfrak{s l}(2, \mathbb{C}))$. There is an alternative way to construct these finite-dimensional representations that shows their close relation to infinite-dimensional representations (see [GMS]). Let us explain how the process goes. It is very similar to the $\mathfrak{s l}(2, \mathbb{C})$ case.

Consider $m=\alpha-\beta$ and $p=\alpha+\beta+1$. Notice that now $C_{\alpha}(m, p) \neq 0$ if $\alpha \in|m|,|m|+1, \ldots, p$, and $C_{p}(m, p)=0$. The underlying vector space for the representation with spins $(\alpha, \beta)$ is $V(m, p)=\stackrel{|m|}{V} \otimes \stackrel{|m|+1}{V} \otimes \cdots \otimes{ }^{p-1} V$, and the explicit form of the representation is given exactly by the same formulae of the infinite-dimensional representations. The equivalence of the representations is a trivial consequence of the Clebsch-Gordan formula. This construction gives us a finite-dimensional representation $\varrho(m, p)$ for each 
pair $(m, p)$ with $m, p \in \mathbb{Z} / 2$ and $p-|m| \in \mathbb{N}_{1}$. It also makes sense for $|p|-|m| \in \mathbb{Z}$, with appropriate changes. As before we have the equivalence $\varrho(m, p) \cong \varrho(-m,-p)$.

Since we completed the sets $W_{m}$ defined at the beginning of 2.1.1, we have a representation $\varrho(m, p)$ of the Lorentz Algebra for each pair $(m, p)$ with $m \in \mathbb{Z} / 2$ and $p \in \mathbb{C}$. All of them have a central character $\lambda_{m, p}$, since the new representations considered are finite-dimensional and irreducible. The finite-dimensional representations give us a framed knot invariant $X_{\text {fin }}(m, p)$ for each pair $m, p \in \mathbb{Z} / 2$ with $|p|-|m| \in \mathbb{N}_{1}$. This invariant is independent of the framing if and only if $m=0$, that is, if $\alpha=\beta$.

Consider now the algebra morphisms $\lambda_{m, p} \circ \phi_{t_{L}}: \mathcal{A} \rightarrow \mathbb{C}$, where $m \in \mathbb{Z}$ and $p \in \mathbb{C}$. The argument is now similar to the one in 1.4.1. If we look at the expression of the representations $\varrho_{m, p}$, it is easy to conclude that given any chord diagram $w$ with $n$ chords, the evaluation $\lambda_{m, p} \circ \phi_{t_{L}}(w)$ is, for fixed $m$, a polynomial in $p$ of degree at most $2 n$. Notice that any factor of the form $C_{\alpha}(m, p)$ appears in the expression for $\lambda_{m, p} \circ \phi_{t_{L}}(w)$ an even number of times. For the case of balanced representations, that is, $m=0$, we can also prove that it is a polynomial in $p^{2}$. Also the value of the polynomials at $p=1$ is zero if $n>0$, for the pair with $m=0$ and $p=1$ yields the trivial one-dimensional representation of $L$. We have proved:

Theorem 11. Consider the framed knot invariants $\left\{X(m, p): m \in \mathbb{N}_{0}\right.$, $p \in \mathbb{C}\}$. If we fix $m \in \mathbb{N}_{0}$ then the term of order $n$ in the expansion of $X(m, p, K)$ as a power series is a polynomial in $p$ of degree at most $2 n$. Here $K$ is any framed knot. If $m=0$ then only the even terms of the expansion are non-zero. Moreover the polynomials are zero at $p=1$ for $n>0$.

Therefore, if we know the value of $X(m, p, K)$ for the finite-dimensional representations, that is, if $|p|-|m| \in \mathbb{N}$, we can determine it for any value of the parameter $p$. This is similar to the $\mathfrak{s l}(2, \mathbb{C})$ case.

2.2.2. Relation to the Coloured Jones Polynomial. The relation between the Lorentz knot invariants that come from finite-dimensional and infinitedimensional representations remarked after Theorem 11 gives us a way to relate the Coloured Jones Polynomial to the Lorentz Group invariants. In fact, we have

THEOREM 12. Let $K$ be some oriented framed knot, and $K^{*}$ its mirror image. Then for any $z, w \in \mathbb{C}$ with $z-w \in \mathbb{Z}$ we have

$$
\frac{J^{z}\left(K^{*}\right)}{2 z+1} \times \frac{J^{w}(K)}{2 w+1}=X(z-w, z+w+1, K)
$$

as formal power series over $\mathbb{C}$.

Proof. For any $m \in \mathbb{Z} / 2$ and $x \in \mathbb{C}$, let $z(x, m)=m+x$ and $w(x, m)=$ $-m+x$. Thus each pair $(z, w) \in \mathbb{C}^{2}$ with $z-w \in \mathbb{Z}$ is of the form 
$(z(x, m), w(x, m))$ for some $m$ and $x$. Fix $m \in \mathbb{Z} / 2$. We want to prove that

$$
\frac{J^{m+x}\left(K^{*}\right)}{2 m+2 x+1} \times \frac{J^{-m+x}(K)}{-2 m+2 x+1}=X(m, 2 x+1, K), \quad \forall x \in \mathbb{C} .
$$

Each term of the formal power series on both sides of the equality is a polynomial in $x$, thus we only need to prove the equality if both $x-m$ and $x+m$ are half integers.

Let $t$ be the infinitesimal $R$-matrix in $\mathfrak{s l}(2, \mathbb{C})$ coming from the CartanKilling form. Notice that it is minus the one considered in 1.4. Let $\alpha$ be a half integer. Recall that for a framed knot $K$ we have

$$
\frac{J^{\alpha}(K)}{2 \alpha+1}=\left(\lambda_{\alpha} \circ \mathcal{Z}_{-t}\right)(K)
$$

Therefore by Theorem 1(iii),

$$
\frac{J^{\alpha}\left(K^{*}\right)}{2 \alpha+1}=\left(\lambda_{\alpha} \circ \mathcal{Z}_{t}\right)(K)
$$

since $\phi_{t}(w)=(-1)^{n} \phi_{-t}(w)$ if $w$ is a chord diagram with $n$ chords.

Let $K$ be a framed knot and $x$ be such that $\alpha=x-m$ and $\beta=x+m$ are half integers. By Theorem 4 we have

$$
\begin{aligned}
\frac{J^{\alpha}\left(K^{*}\right)}{2 \alpha+1} \times \frac{J^{\beta}(K)}{2 \beta+1} & =\left(\lambda_{\alpha} \circ \mathcal{Z}_{t}\right)(K) \times\left(\lambda_{\beta} \circ \mathcal{Z}_{-t}\right)(K) \\
& =\left(\lambda_{\alpha} \circ \mathcal{Z}_{t^{l}}\right)(K) \times\left(\lambda_{\beta} \circ \mathcal{Z}_{-t^{r}}\right)(K) \\
& =\left(\left(\lambda_{\alpha} \otimes \lambda_{\beta}\right) \circ \mathcal{Z}_{t_{L}}\right)(K) .
\end{aligned}
$$

Recall $t_{L}=t^{l}-t^{r}$.

Now, $\lambda_{\alpha} \otimes \lambda_{\beta}$ is the central character of the representation $\varrho_{\alpha} \otimes \varrho_{\beta}$ of $U(L) \cong U(\mathfrak{s l}(2, \mathbb{C})) \otimes U(\mathfrak{s l}(2, \mathbb{C}))$. As we have seen before, this representation is equivalent to $\varrho(\alpha-\beta, \alpha+\beta+1)=\varrho(m, 2 x+1)$. Thus their central characters are the same. This proves

$$
\left(\left(\lambda_{\alpha} \otimes \lambda_{\beta}\right) \circ \mathcal{Z}_{t_{L}}\right)(K)=\left(\lambda_{m, 2 x+1} \circ \mathcal{Z}_{t_{L}}\right)(K)
$$

if both $x-m$ and $x+m$ are half integers, and the proof is finished.

We have the following simple consequences.

Corollary 13. Given a framed knot $K$, the term of order $n$ in the power series of $X(m, z, K)$ is a polynomial in $m$ and $z$.

Corollary 14. If $O$ is the unknot, then $X(m, p, O)$ is a convergent power series.

Conollary 15. For balanced representations, that is, if $m=0$, the invariant $X(0, p)$ does not distinguish a knot from its mirror image.

Corollary 16. The framed knot invariants $X(m, p)$ are unoriented. 


\section{RELATIONS TO THE APPROACH OF BUFFENOIR AND ROCHE}

The aim of this section is to give a sketch of how the Buffenoir and Roche description of the infinite-dimensional unitary representations of the Quantum Lorentz Group relates to our approach. The Quantum Lorentz Group was originally defined by Woronowicz and Podleś in [PoW]. The classification of its irreducible unitary representations appeared first in $[\mathrm{Pu}]$.

For an expanded treatment of the issues considered in this section, we refer the reader to $[\mathrm{PhD}]$.

\subsection{Representations of the Quantum Lorentz Group and $R$ -} matrices. We now follow [BR1]. Other good references are [BR2] and $[\mathrm{BNR}]$. These references contain all the notation and conventions we use. The Quantum Lorentz Group $\mathcal{D}$ at a point $q \in(0,1)$ is defined as the quantum double $\mathcal{D}\left(U_{q}(\mathfrak{s u}(2)), \operatorname{Pol}\left(S U_{q}(2)\right)\right)$. Notice that both $U_{q}(\mathfrak{s u}(2))$ and $\operatorname{Pol}\left(S U_{q}(2)\right)^{\text {cop }}$ are Hopf subalgebras of $\mathcal{D}$. The Quantum Lorentz Group thus has a formal $R$-matrix coming from its quantum double structure. Even though it is defined by an infinite sum, it is possible to describe its action in any pair of infinite-dimensional irreducible representations of $\mathcal{D}$ in the principal series. See [BR1, BR2] for their description. For the dual counterpart of the theory, in other words for the theory of corepresentations of the algebra of functions in the Quantum Lorentz Group $S L_{q}(2, \mathbb{C})$, we refer to $[\mathrm{PuW}]$.

Let us describe what the situation is in the case when the two representations are the same. Suppose also their minimal spin $m$ is zero. Similarly to the classical case described above, representations $\varrho(p)$ of this kind will be called balanced. They depend on a parameter $p \in \mathbb{C}$. If $p \in i \mathbb{R}$ then the representations $\varrho(p)$ can be made unitary. Choosing $p \in[0, i 2 \pi / h]$, where $q=e^{h / 2}$, parametrises all the unitary representations in the principal series which have minimal spin zero. The latter are called simple representations in $[\mathrm{NR}]$.

Similarly to the classical case, the underlying vector space of the balanced representations $\varrho(p), p \in \mathbb{C}$, of the Quantum Lorentz Group is

$$
V=V(p)=\bigoplus_{\alpha \in \mathbb{N}_{0}} \stackrel{\alpha}{V}
$$

where

$$
\stackrel{\alpha}{\varrho}: U_{q}(\mathfrak{s u}(2)) \rightarrow L(\stackrel{\alpha}{V})
$$

is the irreducible representation of $U_{q}(\mathfrak{s u}(2))$ with spin $\alpha$. A basis of $\stackrel{\alpha}{V}$ is thus given by the vectors $\left\{v_{i}^{\alpha}: i=-\alpha,-\alpha+1, \ldots, \alpha\right\}$. Any element $x$ of 
$U_{q}(\mathfrak{s u}(2))$ acts in $V$ as

$$
\prod_{\alpha \in \mathbb{N}_{0}} \stackrel{\alpha}{\varrho}(x)
$$

A group-like element of the Lorentz Group is given by $G=q^{2 J_{z}}$. The heuristic ribbon element of the Quantum Lorentz Group is easily proved to act as the identity in balanced representations. See $[\mathrm{NR}]$.

Given half integers $A, B, C$ and $D$, define the complex numbers

$$
\Lambda_{A D}^{B C}(p)=\sum_{\sigma}\left(\begin{array}{c|cc}
0 & C & B \\
A & \sigma & -\sigma
\end{array}\right) q^{2 \sigma p}\left(\begin{array}{cc|c}
-\sigma & \sigma & D \\
B & C & 0
\end{array}\right) .
$$

For the correct definition of the phases of the Clebsch-Gordan coefficients see [BR2]. We display their explicit expression later. The formal universal $R$-matrix of the Quantum Lorentz Group is (see [BR1])

$$
\mathcal{R}=\sum_{\substack{\alpha \in \frac{1}{2} \mathbb{N}_{0} \\-\alpha \leq i_{\alpha}, j_{\alpha} \leq \alpha}} \stackrel{\alpha}{X}_{j_{\alpha}}^{i_{\alpha}} \otimes \stackrel{\alpha}{g}_{i_{\alpha}}^{j_{\alpha}}
$$

its inverse being

$$
\mathcal{R}^{-1}=\sum_{\substack{\alpha \in \frac{1}{2} \mathbb{N}_{0} \\-\alpha \leq i_{\alpha}, j_{\alpha} \leq \alpha}} \stackrel{\alpha}{X}_{j_{\alpha}}^{i_{\alpha}} \otimes S^{-1}\left(\stackrel{\alpha_{g}^{j_{\alpha}}}{j_{i_{\alpha}}}\right) .
$$

This antipode $S$ is the one of $\operatorname{Pol}\left(S U_{q}(2)\right)^{\text {cop }} \subset \mathcal{D}$, which is the inverse of the one in $\operatorname{Pol}\left(S U_{q}(2)\right)$, thus

$$
S^{-1}\left(\begin{array}{l}
\alpha_{g}^{j_{\alpha}} \\
i_{\alpha}
\end{array}\right)=q^{-i_{\alpha}+j_{\alpha}}(-1)^{-j_{\alpha}+i_{\alpha}} \stackrel{\alpha}{g}_{-j_{\alpha}}
$$

(see $[\mathrm{BR} 2$, equation $(25)])$. The action of $\stackrel{\alpha}{g}_{j_{\alpha}}^{i_{\alpha}}$ in the space $V(p)$ is given by

$$
\begin{aligned}
& \stackrel{\alpha}{g_{i_{\alpha}}} \stackrel{\beta}{v_{i_{\beta}}} \\
& =\frac{\mathcal{F}_{\beta}}{\mathcal{F}_{\gamma}} \sum_{D, \gamma, x \in \frac{1}{2}} \sum_{\mathbb{N}_{0}} \sum_{-\gamma \leq i_{\gamma} \leq \gamma}{\stackrel{\gamma}{v_{i \gamma}}}_{i_{\gamma} \leq x \leq D}\left(\begin{array}{cc|c}
i_{\gamma} & i_{\alpha} & D \\
\gamma & \alpha & x
\end{array}\right)\left(\begin{array}{c|cc}
x & \alpha & \beta \\
D & j_{\alpha} & i_{\beta}
\end{array}\right) \Lambda_{\gamma \beta}^{\alpha C} .
\end{aligned}
$$

Note that this is a finite sum. The constants $\mathcal{F}_{\alpha}$ are defined in [BR2, Proposition 1]. They will not be used directly. In fact their values are (almost) arbitrary and they only appear to ensure that the representations $\varrho(p)$ are unitary for $p \in i \mathbb{R}$ and the natural inner product in $V$. Their appearance does not change the representation itself, therefore does not affect the calculations of knot invariants.

The coefficients $\Lambda_{A D}^{B C}(p)$ are originally defined in [BR2] from an analytic continuation of $6 j$-symbols, and at the end are proved to coincide with (1). 
One can show directly that (2) does define a representation of the Quantum Lorentz Group for any $p \in \mathbb{C}$, since equation (76) of [BR2] holds (see [PhD]).

In some particular cases, equation (2) simplifies to

$$
\stackrel{\alpha}{g}_{j_{\alpha}}^{i_{\alpha}} v_{0}^{0}=\frac{\mathcal{F}_{0}}{\mathcal{F}_{\gamma}} \sum_{\gamma, i_{\gamma}}\left(\begin{array}{cc|c}
i_{\gamma} & i_{\alpha} & \alpha \\
\gamma & \alpha & j_{\alpha}
\end{array}\right) \Lambda_{\gamma 0}^{\alpha \alpha} \hat{v}_{i_{\gamma}}^{\gamma}
$$

and

$$
\left\langle v^{0},{ }^{\alpha}{ }_{i_{\alpha}}^{\beta} v_{i_{\beta}}\right\rangle=\frac{\mathcal{F}_{\beta}}{\mathcal{F}_{0}}\left(\begin{array}{c|cc}
i_{\alpha} & \alpha & \beta \\
\alpha & j_{\alpha} & i_{\beta}
\end{array}\right) \Lambda_{0 \beta}^{\alpha \alpha} .
$$

All these formulae are consequences of well known symmetries of ClebschGordan coefficients listed for example in [BR2]. Using them we can also prove $\Lambda_{\alpha \alpha}^{0 \alpha}=1$, from which it follows that

$$
{ }_{g_{0}^{0}}^{0} v_{i_{\alpha}}^{\alpha}=\stackrel{\alpha}{v_{i_{\alpha}}}
$$

The elements

$$
\stackrel{\alpha}{X}_{j_{\alpha}}^{i_{\alpha}} \in \operatorname{Pol}\left(S U_{q}(2)\right)^{*}, \quad \alpha \in \frac{1}{2} \mathbb{N}_{0}, i_{\alpha}=-\alpha, \ldots, \alpha,
$$

act simply as matrix elements, that is:

$$
\stackrel{\alpha}{X}{ }_{j_{\alpha}}^{i_{\alpha}}{\stackrel{\beta}{i_{\beta}}}_{i_{\beta}}=\delta(\alpha, \beta) \delta\left(i_{\alpha}, i_{\beta}\right) \stackrel{\alpha}{v_{j_{\alpha}}}
$$

Notice $U_{q}(\mathfrak{s u}(2))$ is naturally embedded in $\operatorname{Pol}\left(S U_{q}(2)\right)^{*}$. Moreover any finite-dimensional irreducible representation of $U_{q}(\mathfrak{s u}(2))$ induces one of $\operatorname{span}\left\{\stackrel{\alpha}{X_{j_{\alpha}}^{i_{\alpha}}}\right\} \subset \operatorname{Pol}\left(S U_{q}(2)\right)^{*}$ which has exactly this form (cf. [PoW, Theorem 5.1]).

The action of the group-like element $G$ is

$$
G \stackrel{\alpha}{v_{i_{\alpha}}}=q^{2 i_{\alpha}} \stackrel{\alpha}{v}_{i_{\alpha}}
$$

It is easy to compute how $\mathcal{R}$ acts:

$$
\mathcal{R}\left({\stackrel{\alpha}{v_{i_{\alpha}}}} \otimes{\stackrel{\beta}{v_{i_{\beta}}}}\right)=\sum_{D, x, \gamma, i_{\gamma}, j_{\alpha}}\left(\begin{array}{cc|c}
i_{\gamma} & j_{\alpha} & D \\
\gamma & \alpha & x
\end{array}\right)\left(\begin{array}{c|cc}
x & \alpha & \beta \\
D & i_{\alpha} & i_{\beta}
\end{array}\right) \frac{\mathcal{F}_{\beta}}{\mathcal{F}_{\gamma}} \Lambda_{\gamma \beta}^{\alpha D}\left(\stackrel{\alpha}{v_{j_{\alpha}}} \otimes \gamma_{i_{\gamma}}\right)
$$

(see [BR1, Proposition 13]). The summation range is the obvious one. The action of $\mathcal{R}$ in $V \otimes V$ is thus well defined. Note we are considering the algebraic, rather than topological, tensor product. Moreover $\mathcal{R}$ defines a braid group representation. Denote it by $b \in B(n) \mapsto R_{b} \in L\left(V^{\otimes}\right)$. Here $B(n)$ denotes the $n$-strand braid group and $L\left(V^{\otimes n}\right)$ the vector space of linear maps $V^{\otimes n} \rightarrow V^{\otimes n}$. Notice that the braiding operators $R_{b}$ extend to unitary operators if $p \in i \mathbb{R}$ since $\mathcal{R}^{* \otimes *}=\mathcal{R}^{-1}$, where $*$ is the star structure on the Quantum Lorentz Group (see [BNR]), because $\varrho(p)$ is unitary in this case. 
3.2. Associated knot invariants. We now use the framework just introduced to define quantum lorentzian knot invariants. As we will see, they relate to our approach before.

3.2.1. Some heuristics. Let $q \in(0,1)$ and $p \in \mathbb{C}$. Suppose we are given a braid $b$ with $n+1$ strands. There is a map $R_{b}: V^{\otimes(n+1)} \rightarrow V^{\otimes(n+1)}$ attached to it. Consider the map $A_{b}=(\mathrm{id} \otimes G \otimes \cdots \otimes G) R_{b}$. Suppose the closure of the braid $b$ is a knot. If the representations we are considering were finite-dimensional, then the partial trace $T^{1}\left(A_{b}\right): V \rightarrow V$ of $A_{b}$ over the last $n$ variables would be an intertwiner and thus a multiple of the identity, since the representations we are considering are irreducible. Moreover this multiple of the identity would be a knot invariant, which would have the form

$$
=\sum_{\substack{\alpha_{1}, \ldots, \alpha_{n} \in \frac{1}{2} \mathbb{N}_{0} \\-\alpha_{k} \leq i_{\alpha_{k}} \leq \alpha_{k}, k=1, \ldots, n}}\left\langle v^{0} \otimes \stackrel{\alpha}{v}^{\alpha_{1}} i_{\alpha_{1}} \otimes \cdots \otimes \stackrel{\alpha_{n}}{v} i_{\alpha_{n}}, A_{b}\left(\stackrel{0}{v}_{0} \otimes \stackrel{\alpha_{1}}{v} i_{i_{\alpha_{1}}} \otimes \cdots \otimes \stackrel{\alpha_{n}}{v} i_{i_{n}}\right)\right\rangle .
$$

Even though the sums above may not be convergent, the assignment of one sum of this kind to a braid whose closure is a knot is not ambiguous. In fact, suppose $b$ has $m+1$ strands and $n$ crossings. We can always express this sum in a more suggestive way, namely as

$$
S_{b}(q, p)=\sum_{\substack{a_{1}, \ldots, \alpha_{n} \\-\alpha_{k} \leq i_{k}, j_{k} \leq \alpha_{k}, k=1, \ldots, n}}\left\langle v^{0}, \prod_{l=1}^{2 n+m} T(\underline{\alpha}, \underline{i}, \underline{j}, l) \stackrel{0}{v}_{0}\right\rangle,
$$

where if $\underline{\alpha}=\left(\alpha_{1}, \ldots, \alpha_{n}\right), \underline{i}=\left(i_{1}, \ldots, i_{n}\right)$ and $\underline{j}=\left(j_{1}, \ldots, j_{n}\right)$, then $T(\underline{\alpha}, \underline{i}, \underline{j}, l)$ can be either a term of the form $\stackrel{\alpha}{g}_{j}$ or $\stackrel{\alpha}{X}_{j}^{i}$ for some $k \in\{1, \ldots, n\}$, or $G$; and moreover for any $k$ there exists an $l$ such that $T(\underline{\alpha}, \underline{i}, \underline{j}, l)$ is an $X_{j}^{i}$, and the same for $\stackrel{\alpha}{g}_{j}$. The two examples below should clarify what we mean. We obviously need to suppose that the closure of $b$ is a knot for this to hold. Notice that the transition from (8) to (9) is totally clear if the representations are finite-dimensional. We take (9) as the definition of $S_{b}(q, p)$ if $b$ is a braid whose closure is a knot.

Let us look at the sums above in a bit more detail. We consider the left and right handed trefoil knots displayed in Figure 6. Call the two braids we have chosen to represent them $T_{+}$and $T_{-}$. The sum for the right handed trefoil knot is

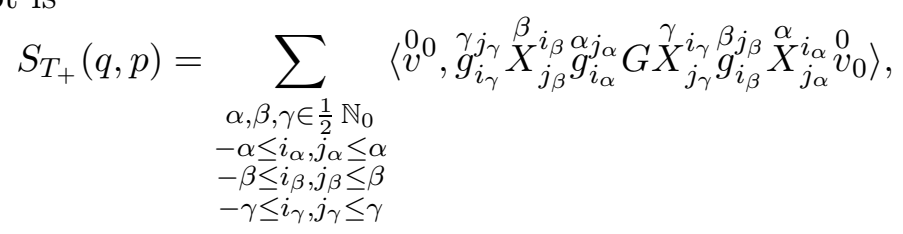



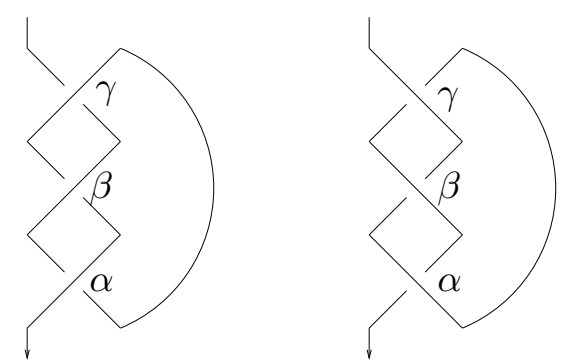

Fig. 6. Right and left handed trefoil knots

whereas for the left handed trefoil it is

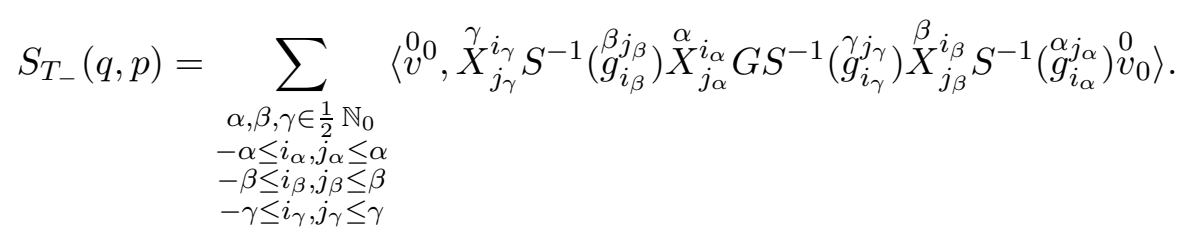

Many of the terms will be zero in the expressions above. Let us look at $S_{T_{-}}$.

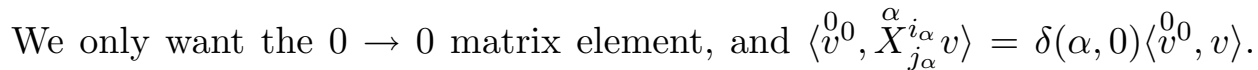
Thus we can make $\gamma=0$, and then note that ${ }_{0}^{0} 0$ acts as the identity. We obtain (we skip unnecessary indices)

$$
\begin{aligned}
& S_{T_{-}}(q, p) \\
& =\sum_{\alpha, \beta \in \frac{1}{2} \mathbb{N}_{0}} q^{-i_{\alpha}+j_{\alpha}-i_{\beta}+j_{\beta}}(-1)^{i_{\alpha}+i_{\beta}-j_{\alpha}-j_{\beta}}\left\langle\stackrel{\beta}{g_{-j_{\beta}}} \stackrel{\alpha}{X} j_{j_{\alpha}}^{i_{\alpha}} G \underset{X_{\beta}}{i_{\beta} g_{-j_{\alpha}}^{\alpha-i_{\alpha}}} \stackrel{0}{v}_{0}, \stackrel{0}{v_{0}}\right\rangle .
\end{aligned}
$$

From (6) and (7) it follows that $\alpha=\beta$ and $i_{\alpha}=j_{\beta}$. By (3) and (4) we conclude that

$$
\begin{aligned}
S_{T_{-}}(q, p)=\sum_{\alpha \in \frac{1}{2} \mathbb{N}_{0} i_{\beta}, j_{\beta}, j_{\alpha}=-\alpha} \sum^{\alpha} q^{j_{\alpha}-i_{\beta}+2 j_{\beta}}(-1)^{i_{\beta}-j_{\beta}} & \\
& \times\left(\begin{array}{c|cc}
-i_{\beta} & \alpha & \alpha \\
\alpha & -j_{\beta} & j_{\alpha}
\end{array}\right)\left(\begin{array}{cc|c}
i_{\beta} & -j_{\beta} & \alpha \\
\alpha & \alpha & -j_{\alpha}
\end{array}\right) \Lambda_{0 \alpha}^{\alpha \alpha} \Lambda_{\alpha 0}^{\alpha \alpha} .
\end{aligned}
$$

Using the standard symmetries of the Clebsch-Gordan coefficients, we can express this as

$$
\begin{aligned}
& S_{T_{-}}(q, p) \\
& =\sum_{\alpha \in \mathbb{N}_{0}} \sum_{i_{\beta}, j_{\beta}, j_{\alpha}=-\alpha}^{\alpha} q^{2 j_{\beta}}\left(\begin{array}{cc|c}
-i_{\beta} & -j_{\alpha} & \alpha \\
\alpha & \alpha & -j_{\beta}
\end{array}\right)\left(\begin{array}{c|cc}
-j_{\beta} & \alpha & \alpha \\
\alpha & -i_{\beta} & -j_{\alpha}
\end{array}\right) \Lambda_{0 \alpha}^{\alpha \alpha} \Lambda_{\alpha 0}^{\alpha \alpha} .
\end{aligned}
$$

Notice that $\Lambda_{\alpha 0}^{\alpha \alpha}$ is zero unless $\alpha$ is an integer. Therefore the final expression 
for the sum is

$$
S_{T_{-}}(q, p)=\sum_{\alpha \in \mathbb{N}_{0}} d_{\alpha} \Lambda_{0 \alpha}^{\alpha \alpha} \Lambda_{\alpha 0}^{\alpha \alpha}
$$

Here $d_{a}$ is the quantum dimension of the representation $\stackrel{\alpha}{\varrho}$, which equals $\left(q^{2 \alpha+1}-q^{-2 \alpha-1}\right) /\left(q-q^{-1}\right)$. This last sum is easily proved to be equal to $S_{T_{+}}$, therefore, if the sums do define a knot invariant, they make no distinction between the trefoil and its mirror image. We would expect this from Corollary 15. The calculations for other knot diagrams follow the same procedure, which can be represented by an obvious graphical calculation.

Notice that the series $S\left(T_{-}\right)$seems to be divergent due to the presence of the $d_{\alpha}$ term. Therefore these sums do not seem to define $\mathbb{C}$-valued knot invariants. This tells us that the method of Borel re-summation sketched in $[\mathrm{FM}]$ is perhaps more powerful.

3.2.2. Finite-dimensional representations. Let $Y(\alpha, \beta, \gamma)=1$ if $\stackrel{\alpha}{\varrho}$ appears in the decomposition of $\varrho \otimes \varrho$ in terms of irreducible representations of $U_{q}(\mathfrak{s u}(2))$ and zero otherwise, where $\alpha, \beta, \gamma \in \frac{1}{2} \mathbb{Z}$. Let also $Y\left(\alpha, i_{\alpha}\right)=1$ if $i_{\alpha} \in\{-\alpha, \ldots, \alpha\}$ and zero otherwise. We have (see [BR2])

$$
\begin{gathered}
\left(\begin{array}{cc|c}
m & n & K \\
I & J & p
\end{array}\right)=Y(I, m) Y(J, n) Y(K, p) \delta(m+n, p) Y(I, J, K) \\
\times q^{m(p+1)+\frac{1}{2}(J(J+1)-I(I+1)-K(K+1))} e^{i \pi(I-m)} \\
\times \sqrt{\frac{[2 K+1][I+J-K] ![I-m] ![J-n] ![K-p] ![K+p] !}{[K+J-I] ![I+K-J] ![I+J+K+1] ![I+m] ![J+n] !}} \\
\times \quad \sum_{-J+K-m \leq V \leq I-m}^{K-p} \frac{q^{V(K+p+1)} e^{i \pi V}[I+m+V] ![J+K-m-V] !}{[V] ![K-p-V] ![I-m-V] ![J-K+m+V] !}
\end{gathered}
$$

Let $p \in \mathbb{C}$. We thus have an infinite-dimensional representation $\varrho(p)$ of the Quantum Lorentz Group given by the constants $\Lambda_{A D}^{B C}(p)$. Its representation space is by definition $V=V(p)=\bigoplus_{\alpha \in \mathbb{N}_{0}} \stackrel{\alpha}{V}$. From equation (10), we can easily calculate the coefficients $\Lambda_{A D}^{B C}$ if $B=1 / 2$ :

Lemma 17. Let $C \geq 0$ be an integer. We have

$$
\begin{array}{ll}
\Lambda_{C C}^{1 / 2, C-1 / 2}(p)=\frac{q^{C}\left(q^{p}+q^{-p}\right)}{q^{2 C}+1}, & \Lambda_{C, C+1}^{1 / 2, C+1 / 2}(p)=\frac{q^{2 C+2} q^{p}-q^{-p}}{q^{2 C+2}+1}, \\
\Lambda_{C C}^{1 / 2, C+1 / 2}(p)=-\frac{q^{C+1}\left(q^{p}+q^{-p}\right)}{q^{2 C+2}+1}, & \Lambda_{C+1, C}^{1 / 2, C+1 / 2}(p)=\frac{q^{2 C+2} q^{-p}-q^{p}}{q^{2 C+2}+1} .
\end{array}
$$


Notice that all the other $\Lambda_{A D}^{B C}(p)$ coefficients with $B=1 / 2$ are zero. See also [BR2, proof of Theorem 3].

In particular, if $p \in \mathbb{N}$, then the representation $\varrho(p)$ has a finite-dimensional subrepresentation $\varrho(p)_{\text {fin }}$ in $V(p)_{\text {fin }}=\stackrel{0}{V} \otimes \stackrel{1}{V} \oplus \ldots \oplus \stackrel{p-1}{V}$. Compare with 2.2.1. Using Schur's lemma as in [BR2, proof of Theorem 3], one proves these representations are irreducible.

Notice that $\stackrel{0}{v}_{0} \in V(p)_{\text {fin. }}$. Therefore, looking at (9) we obtain

Lemma 18. If $p \in \mathbb{N}$ and $b$ is a braid whose closure is a knot, then the infinite sum $S_{b}(q, p)$ reduces to a finite sum for any $q \in(0,1)$.

As mentioned in the introduction, the category of finite-dimensional representations of the Quantum Lorentz Group is (almost) ribbon equivalent to the category of finite-dimensional representations of $U_{q}(\mathfrak{s u}(2)) \otimes_{R^{-1}}$ $U_{q}(\mathfrak{s u}(2))$ where $R$ is the $R$-matrix of $U_{q}(\mathfrak{s u}(2))$. Let us explain what this means. We follow [BR2] and [BNR] closely. The ribbon Hopf algebra $U_{q}(\mathfrak{s u}(2)) \otimes_{R^{-1}} U_{q}(\mathfrak{s u}(2))$ is isomorphic to $U_{q}(\mathfrak{s u}(2)) \otimes U_{q}(\mathfrak{s u}(2))$ as an algebra, but has a coalgebra structure of the form

$$
\Delta(a \otimes b)=R_{23}^{-1} a^{\prime} \otimes b^{\prime} \otimes a^{\prime \prime} \otimes b^{\prime \prime} R_{23},
$$

whereas the antipode is defined as

$$
S(a \otimes b)=R_{21} S(a) \otimes S(b) R_{21}^{-1} .
$$

This Hopf algebra has an $R$-matrix given by

$$
\widehat{R}=R_{14}^{(-)} R_{24}^{(-)} R_{13}^{(+)} R_{23}^{(+)},
$$

where $R^{(+)}=R$ and $R^{(-)}=R_{21}^{-1}$. The algebra $U_{q}(\mathfrak{s u}(2)) \otimes_{R^{-1}} U_{q}(\mathfrak{s u}(2))$ is a ribbon Hopf algebra with group-like element $G \otimes G$, where $G=q^{2 J_{z}}$ is the group-like element of $U_{q}(\mathfrak{s u}(2))$ (see [BNR, p. 4969]).

The irreducible finite-dimensional representations $\varrho_{w}$ of $U_{q}(\mathfrak{s u}(2))$ are parametrised by $\alpha \in \frac{1}{2} \mathbb{N}_{0}$ and $w \in\{1,-1, i,-i\}$, the level of the representation (see $[\mathrm{KS}$, Theorem 13]). The irreducible representations $\stackrel{\alpha}{\varrho}$ of spin $\alpha$ are the ones for which $w=1$. They are the natural quantisation of the representations of $S U(2)$ of $\operatorname{spin} \alpha$. The action of the $R$-matrix of $U_{q}(\mathfrak{s u}(2))$ is, a priori, only defined on pairs of representations of level 1 , or their direct sums. Nevertheless, the category of finite-dimensional representations of $U_{q}(\mathfrak{s u}(2))$ of this kind is a ribbon category. Let $\stackrel{\alpha}{\varrho}$ and $\stackrel{\beta}{\varrho}$ be two finite-dimensional irreducible representations of $U_{q}(\mathfrak{s u}(2))$ of level 1 which will then generate a representation $\stackrel{\alpha}{\varrho} \otimes \stackrel{\beta}{\varrho}$ of $U_{q}(\mathfrak{s u}(2)) \otimes_{R^{-1}} U_{q}(\mathfrak{s u}(2))$. The action of the $R$-matrix of $U_{q}(\mathfrak{s u}(2)) \otimes_{R^{-1}} U_{q}(\mathfrak{s u}(2))$ is well defined on pairs of representations of this kind. The same is true for the group-like element, thus we 
can define a framed knot invariant $I\left(\stackrel{\alpha}{\varrho} \otimes \varrho^{\beta}\right)$. Unpacking its expression yields immediately

LEMMA 19. For any framed knot $K$ we have

$$
I(\stackrel{\alpha}{\varrho} \otimes \stackrel{\beta}{\varrho})(K)=I(\stackrel{\alpha}{\varrho})\left(K^{*}\right) I(\stackrel{\beta}{\varrho})(K)
$$

where $K^{*}$ is the mirror image of $K$. Here $I(\stackrel{\alpha}{\varrho})$ is the $U_{q}(\mathfrak{s u}(2))$-framed knot invariant defined from $\stackrel{\alpha}{\varrho}$, in other words the Coloured Jones Polynomial, and the same for $I(\varrho)$.

There exists a Hopf algebra morphism $\psi: \mathcal{D} \rightarrow U_{q}(\mathfrak{s u}(2)) \otimes_{R^{-1}} U_{q}(\mathfrak{s u}(2))$ of the form

$$
\psi:(x, f) \mapsto \sum_{(x)(f)} x^{\prime}\left(f^{\prime \prime} \otimes \mathrm{id}\right)\left(R^{(+)}\right) \otimes x^{\prime \prime}\left(f^{\prime} \otimes \mathrm{id}\right)\left(R^{(-)}\right) .
$$

The comultiplications are taken in $U_{q}(\mathfrak{s u}(2))$ and $\operatorname{Pol}\left(S U_{q}(2)\right)$. This morphism naturally extends to the elements $\stackrel{\alpha}{X}_{j_{\alpha}}^{i_{\alpha}} \in \operatorname{Pol}\left(S U_{q}(2)\right)^{*}$.

There also exists a morphism $s: \operatorname{Pol}\left(S U_{q}(2)\right) \rightarrow \operatorname{Pol}\left(S U_{q}(2)\right)$ such that $s\left(\begin{array}{l}\alpha_{i_{\alpha}} \\ j_{\alpha}\end{array}\right)=(-1)^{2 \alpha} g_{j_{\alpha}}^{\alpha_{i_{\alpha}}}$. It extends to all the Quantum Lorentz Group provided we define its restriction to $U_{q}(\mathfrak{s u}(2))$ (thus also to $\operatorname{span}\left\{\stackrel{\alpha}{X_{j_{\alpha}}^{i_{\alpha}}}\right\} \subset$ $\left.\operatorname{Pol}\left(S U_{q}(2)\right)^{*}\right)$ to be the identity. The main result of [T, Theorem 5.4], is the following:

THEOREM 20. Let $\varrho$ be an irreducible finite-dimensional representation of the Quantum Lorentz Group $\mathcal{D}$ which has a structure of a $\operatorname{Pol}\left(S U_{q}(2)\right)$ crossed bimodule. In our case this means that the representation @ restricted to $U_{q}(\mathfrak{s u}(2))$ is a direct sum of representations $\stackrel{\alpha}{\varrho_{w}}$ with $\omega=1$, which is what happens for the representations $\varrho(p)_{\mathrm{fin}}, p \in \mathbb{N}$ (see [T, Proposition 5.1]); (these representations define corepresentations of the algebra $S_{q} L(2, \mathbb{C})$, thus representations of the Quantum Lorentz Group in the sense of [PoW]). Then there exist $\alpha, \beta \in \frac{1}{2} \mathbb{N}_{0}$ such that either $\varrho=(\stackrel{\alpha}{\varrho} \otimes \stackrel{\beta}{\varrho}) \circ \psi$ or $\varrho=\left(\begin{array}{l}\alpha \\ \varrho\end{array} \stackrel{\beta}{\varrho}^{\varrho}\right) \circ \psi \circ s$.

See [BR2, p. 507]. Therefore if $p \in \mathbb{N}$ and $\alpha=(p-1) / 2$, then either $\varrho(p)_{\text {fin }}=(\stackrel{\alpha}{\varrho} \otimes \stackrel{\alpha}{\varrho}) \circ \psi$ or $\varrho(p)_{\text {fin }}=\left(\stackrel{\alpha}{\varrho} \otimes \stackrel{\alpha}{\varrho}_{i}\right) \circ \psi \circ s$, since the minimal spin of $\varrho(p)_{\text {fin }}$ is zero (note that $\psi$ restricted to $U_{q}(\mathfrak{s u}(2))$ is simply the coevaluation $\Delta)$. With a bit more work one can actually prove that the latter case holds (see $[\mathrm{PhD}])$.

If we consider the action in finite-dimensional representations of the form $\varrho=(\stackrel{\alpha}{\varrho} \otimes \stackrel{\beta}{\varrho}) \circ \psi$, then $\psi$ transforms the $R$-matrix of the Quantum Lorentz Group into the $R$-matrix of $U_{q}(\mathfrak{s u}(2)) \otimes_{R^{-1}} U_{q}(\mathfrak{s u}(2))$, and analogously for their inverses. This is an easy consequence of the fact that $(\Delta \otimes \mathrm{id})(R)=R_{13} R_{23}$ and $(\mathrm{id} \otimes \Delta)(R)=R_{13} R_{12}$. The same is true for the 
balanced representations $\varrho(p)_{\text {fin }} \cong(\stackrel{\alpha}{\varrho} \otimes \stackrel{\alpha}{\varrho}) \circ \psi \circ s$ since given that $\stackrel{\alpha}{X}_{j_{\alpha}}^{i_{\alpha}} \otimes \stackrel{\alpha}{g}_{j_{\alpha}}^{j_{\alpha}}$ acts as zero in $V(p)_{\text {fin }} \otimes V(p)_{\text {fin }}$ if $\alpha \in \mathbb{N}+1 / 2$, it follows that the actions of $(s \otimes s)(\mathcal{R})$ and $\mathcal{R}$ in $V(p)_{\text {fin }} \otimes V(p)_{\text {fin }}$ are the same. The detailed calculation appears in $[\mathrm{PhD}]$. The map $\psi$ preserves the group-like elements since $\Delta\left(q^{2 J_{z}}\right)=q^{2 J_{z}} \otimes q^{2 J z}$. Therefore from Lemma 19 we obtain:

Proposition 21. Let $q \in(0,1)$ and $p \in \mathbb{N}$. Let also $\alpha=(p-1) / 2$. Given $a$ braid $b$, let $K_{b}$ be the closure of $b$ with an arbitrary framing and $K_{b}^{*}$ its mirror image. Suppose $K_{b}$ is a knot. Then

$$
S_{b}(q, p)=\frac{I(\varrho)\left(K_{b}^{*}\right) I(\stackrel{\alpha}{\varrho})\left(K_{b}\right)}{[2 \alpha+1]^{2}}=X\left(0, p, K_{b}\right)(h) \frac{(2 \alpha+1)^{2}}{[2 \alpha+1]^{2}},
$$

where $q=\exp (h / 2)$. The last equality follows from Theorem 12 .

Recall also Lemma 18 and notice that $X\left(0, p, K_{b}\right)(h)$ is a convergent power series if $p \in \mathbb{N}$. Therefore the perturbative framework of the previous sections is correct, at least for finite-dimensional representations. In the following we will generalise this to infinite-dimensional representations.

3.2.3. The series are convergent $h$-adically. We now define the $h$-adic version of the theory developed by Buffenoir and Roche. Let $q \in(0,1)$ and consider the element ${ }_{j_{\alpha}}^{\alpha_{\alpha_{\alpha}}} \in \operatorname{Pol}\left(S U_{q}(2)\right)$. For any $p \in \mathbb{C}$, we have a balanced representation $\varrho(p)$ of the Quantum Lorentz Group in $V(p)$. The term

$$
\left\langle\beta^{i_{\beta}}\left|\varrho(p)\left({\stackrel{\alpha}{i_{\alpha}}}_{j_{\alpha}}\right)\right| \hat{v}_{i_{\gamma}}\right\rangle_{q}
$$

can be seen as a function of $q$. Since the building blocks of $\varrho(p)$ are ClebschGordan coefficients, it can be expressed as a sum of square roots of rational functions of $q$, which extends to a well defined analytic function in a neighbourhood of 1 . We can see this for example from (10). In addition we have some terms of the form $q^{p \sigma}, \sigma \in \mathbb{Z}$, which after putting $q=\exp (h / 2)$ define an analytic function of $h$. Therefore

$$
h \mapsto\left\langle{ }^{\beta} i^{i_{\beta}}\left|\varrho(p)\left(\begin{array}{l}
\alpha_{i_{\alpha}} \\
j_{\alpha}
\end{array}\right)\right| \hat{v}_{i_{\gamma}}^{\gamma}\right\rangle_{\exp (h / 2)}
$$

defines a unique power series in $h$. In particular it follows that if $b$ is a braid then each term of the sum $S_{b}(\exp (h / 2), p)$ defines uniquely a power series in $h$, which converges to the term for $h$ small enough.

Lemma 22. For any $x \in \operatorname{Pol}\left(S U_{q}(2)\right)$, the order of

$$
h \mapsto\left\langle v^{\beta_{\beta}}|\varrho(p)(x)| v_{i_{\gamma}}\right\rangle_{\exp (h / 2)},
$$

as a power series in $h$, is greater than or equal to $|\beta-\gamma|$.

Proof. Notice that $\stackrel{\frac{1}{2}}{g}_{j}^{i}$ sends $\stackrel{\gamma}{V}$ to $\stackrel{\gamma-1}{V} \oplus \stackrel{\gamma}{V} \oplus \stackrel{\gamma+1}{V}$ in such a way that for $q=1$ the projection $v$ of $\stackrel{\frac{1}{2}}{g}_{j}^{i} v_{i_{\gamma}}$ in $\stackrel{\gamma+1}{V} \oplus \stackrel{\gamma-1}{V}$ is zero. We can see this from 
Lemma 17. In particular $v$ has order at least one. This lemma is thus a trivial consequence of the fact that the elements $\left\{\stackrel{1}{2}_{j}^{\frac{1}{g}}:-1 / 2 \leq i, j \leq 1 / 2\right\}$ generate $\operatorname{Pol}\left(S U_{q}(2)\right)$ as an algebra.

Proposition 23. For any braid $b$ whose closure is a knot the infinite sum $S_{b}(\exp (h / 2), p)$ converges in the $h$-adic topology.

Proof. Let $b$ be a braid with $n$ crossings and $m+1$ strands. Recall equation (9) and comments after it. Due to the way the ${ }^{\alpha_{k}}{ }_{j}^{i}$ as well as $G$ act in $V(p)$, the previous lemma guarantees that the order of the scalar product $\left\langle v^{0}, \prod_{l=1}^{2 n+m} T(\underline{\alpha}, \underline{i}, \underline{j}, l) \hat{v}_{0}^{0}\right\rangle$ as a power series in $h$ is at least $\alpha_{k}$, for $k=1, \ldots, n$; and the result follows.

3.2.4. The series define a $\mathbb{C}[[h]]$-valued knot invariant. Since we have proved the $h$-adic convergence of the sums $S_{b}(\exp (h / 2), p)$ to a formal power series, we could now use Markov's theorem and prove that the assignment $b \mapsto S_{b}(\exp (h / 2), p)$ defines a knot invariant. However, the best way to prove this is to reduce it to the finite-dimensional case, since we already know that it defines a knot invariant and the exact form of it (see Proposition 21). Consider a coefficient $\Lambda_{A D}^{B C}(p)_{q}$ at $q=\exp (h / 2)$; it is a power series in $h$ convergent for $h$ small enough. From equation (1), we can see that the dependence of each term on $p$ is polynomial. In particular, we have:

LEMma 24. Let $b$ be a braid; consider the power series $S_{b}(\exp (h / 2), p)$ as a function of $p$, the parameter defining a balanced representation of the Quantum LorentzGroup. Then each term in the expansion of $S_{b}(\exp (h / 2), p)$ as a power series in $h$ is a polynomial in $p$.

Proof. Suppose $A(p)=\sum_{n \in \mathbb{N}_{0}} A_{n}(p) h^{n}$ and $B(p)=\sum_{n \in \mathbb{N}_{0}} B_{n}(p) h^{n}$ are power series whose coefficients depend polynomially on $p$, for example power series such as $\exp (m p h / 2)$. Then also the coefficients of their product depend polynomially on $p$. This immediately proves this lemma. Note that the Clebsch-Gordan coefficients as well as the actions of $G$ and of the elements $\stackrel{\alpha}{X}_{j_{\alpha}}^{i_{\alpha}}$ do not depend on $p$.

TheOREm 25. Let $p \in \mathbb{C}$ and $b$ be a braid whose closure is a knot. Let $K$ be the closure of $b$. Then

$$
S_{b}(\exp (h / 2), p)=\frac{X(0, p, K)(2 \alpha+1)^{2}}{[2 \alpha+1]^{2}}
$$

where $\alpha=(p-1) / 2$.

Recall that by Theorem 10 the knot invariant $X(0, p)$ is unframed.

Proof. By Lemma 24, we only need to prove this theorem for $p \in \mathbb{N}$. In this case, if $q=(0,1)$ then $S_{b}(q, p)$ reduces to a finite sum which from 
Proposition 21 equals $X(0, p, K)(2 \alpha+1)^{2}[2 \alpha+1]^{-2}$ at $q=\exp (h / 2)$. Recall that this power series is convergent if $p$ is an integer. Each term of the finite sum $S_{b}(\exp (h / 2), p)$ is a power series in $h$ convergent for $h$ small enough and coinciding with $S_{b}(q, p)$ for $q \in(0,1)$ and close enough to 1 ; thus the result follows.

Acknowledgements. This work was realised in the course of my $\mathrm{PhD}$ in the University of Nottingham under the supervision of Dr. John W. Barrett. I was financially supported by the programme "PRAXIS-XXI", grant number SFRH/BD/1004/2000 of Fundação para a Ciência e a Tecnologia (FCT), financed by the European Community fund Quadro Comunitário de Apoio III, and also by Programa Operacional "Ciência, Tecnologia, Inovação" (POCTI) of the Fundação para a Ciência e a Tecnologia (FCT), cofinanced by the European Community fund FEDER. The last stage of this work was financed by the FCT post-doc grant $S F R H / B D P / 17552 / 2004$, part of the research project POCIT/MAT/60352/2004 ("Quantum Topology").

\section{References}

[AC] D. Altschuler and A. Coste, Quasi-quantum groups, knots, three manifolds and topological field theory, Comm. Math. Phys. 150 (1992), 83-107.

[B] D. Bar-Natan, On the Vassiliev knot invariants, Topology 34 (1995), 423-472.

[BG] D. Bar-Natan and S. Garoufalidis, On the Melvin-Morton-Rozansky conjecture, Invent. Math. 125 (1996), 103-133.

[BLT] D. Bar-Natan, T. Q. T. Le and D. Thurston, Two applications of elementary knot theory to Lie algebras and Vassiliev invariants, Geom. Topol. 7 (2003), 1-31.

$[\mathrm{BC}] \mathrm{J} . \mathrm{W}$. Barrett and L. Crane, Relativistic spin networks and quantum gravity, J. Math. Phys. 39 (1998), 3296-3302.

[BNR] E. Buffenoir, K. Noui and Ph. Roche, Hamiltonian quantization of Chern-Simons theory with $\mathrm{SL}(2, \mathbb{C})$ group, Classical Quantum Gravity 19 (2002), 4953-5015.

[BR1] E. Buffenoir and Ph. Roche, Tensor product of principal unitary representations of quantum Lorentz group and Askey-Wilson polynomials, J. Math. Phys. 41 (2000), 7715-7751.

[BR2] - - - Harmonic analysis on the quantum Lorentz group, Comm. Math. Phys. 207 (1999), 499-555.

[CP] V. Chari and A. Pressley, A Guide to Quantum Groups, Cambridge Univ. Press, Cambridge, 1994.

[C] S. Chmutov, A proof of the Melvin-Morton conjecture and Feynman diagrams, J. Knot Theory Ramif. 7 (1998), 23-40.

[CS] S. Chmutov and S. Duzhin, The Kontsevich integral, Acta Appl. Math. 66 (2001), 155-190.

[CV] S. Chmutov and A. Varchenko, Remarks on the Vassiliev invariants coming from $\mathfrak{s l}_{2}$, Topology 36 (1997), 153-178.

[D] V. G. Drinfeld, Quasi-Hopf algebras, Algebra i Analiz 1 (1989), no. 6, 114-148 (in Russian); English transl.: Leningrad Math. J. 1 (1990), 1419-1457. 
[FM] J. Faria Martins, On the analytic properties of the $z$-coloured Jones polynomial, QA/0310394, to appear in J. Knot Theory Ramif.

[PhD] -, Quantum topology and the Lorentz group, PhD thesis, Univ. of Nottingham, 2004.

[GMS] I. M. Gel'fand, R. A. Minlos and Z. Ya. Shapiro, Representations of the Rotation and Lorentz Groups and Their Applications, Pergamon Press, Oxford, 1963.

[G] S. Gukov, Three-dimensional quantum gravity, Chern-Simons theory, and the A-polynomial, Comm. Math. Phys. 255 (2005), 577-627.

[K] C. Kassel, Quantum Groups, Grad. Texts in Math. 155, Springer, New York, 1995.

[Kir] A. A. Kirillov, Elements of the Theory of Representations, Grundlehren Math. Wiss. 220, Springer, Berlin, 1976.

[KS] A. Klimyk and K. Schmüdgen, Quantum Groups and Their Representations, Texts and Monographs in Physics, Springer, Berlin, 1997.

[L] S. Lang, $S L(2 ; \mathbb{R})$, Addison-Wesley, Reading, MA, 1975.

[LM] T. Q. T. Le and J. Murakami, The universal Vassiliev-Kontsevich invariant for framed oriented links, Compos. Math. 102 (1996), 41-64.

[MM] P. M. Melvin and H. R. Morton, The coloured Jones function, Comm. Math. Phys. 169 (1995), 501-520.

[NR] K. Noui and Ph. Roche, Cosmological deformation of Lorentzian spin foam models, Classical Quantum Gravity 20 (2003), 3175-3213.

[PoW] P. Podleś and S. L. Woronowicz, Quantum deformation of Lorentz group, Comm. Math. Phys. 130 (1990), 381-431.

$[\mathrm{Pu}] \quad$ W. Pusz, Irreducible unitary representations of quantum Lorentz group, ibid. 152 (1993), 591-626.

$[\mathrm{PuW}]$ W. Pusz and S. L. Woronowicz, Representations of quantum Lorentz group on Gelfand spaces, Rev. Math. Phys. 12 (2000), 1551-1625.

[T] M. Takeuchi, Finite-dimensional representations of the quantum Lorentz group, Comm. Math. Phys. 144 (1992), 557-580.

[V] V. S. Varadarajan, Lie Groups, Lie Algebras, and Their Representations, Grad. Texts in Math. 102, Springer, New York, 1984.

[W] S. Willerton, The Kontsevich integral and algebraic structures on the space of diagrams, in: Knots in Hellas '98 (Delphi), Ser. Knots Everything 24, World Sci., River Edge, NJ, 2000, 530-546.

Departamento de Matemática

Instituto Superior Técnico

Av. Rovisco Pais

1049-001 Lisboa, Portugal

E-mail: jmartins@math.ist.utl.pt

Received 25 March 2004;

in revised form 3 May 2005 